\title{
LIMITS OF MULTIPOLE PLURICOMPLEX GREEN FUNCTIONS
}

\author{
JÓN I. MAGNÚSSON, ALEXANDER RASHKOVSKII, RAGNAR \\ SIGURDSSON, PASCAL J. THOMAS
}

\begin{abstract}
Let $\Omega$ be a bounded hyperconvex domain in $\mathbb{C}^{n}, 0 \in$ $\Omega$, and $S_{\varepsilon}$ a family of $N$ poles in $\Omega$, all tending to 0 as $\varepsilon$ tends to 0 . To each $S_{\varepsilon}$ we associate its vanishing ideal $\mathcal{I}_{\varepsilon}$ and pluricomplex Green function $G_{\varepsilon}=G_{\mathcal{I}_{\varepsilon}}$.

Suppose that, as $\varepsilon$ tends to $0,\left(\mathcal{I}_{\varepsilon}\right)_{\varepsilon}$ converges to $\mathcal{I}$ (local uniform convergence), and that $\left(G_{\varepsilon}\right)_{\varepsilon}$ converges to $G$, locally uniformly away from 0 ; then $G \geq G_{\mathcal{I}}$. If the Hilbert-Samuel multiplicity of $\mathcal{I}$ is strictly larger than its length (codimension, equal to $N$ here), then $\left(G_{\varepsilon}\right)_{\varepsilon}$ cannot converge to $G_{\mathcal{I}}$. Conversely, if $\mathcal{I}$ is a complete intersection ideal, then $\left(G_{\varepsilon}\right)_{\varepsilon}$ converges to $G_{\mathcal{I}}$. We work out the case of three poles.
\end{abstract}

\section{Contents}

1. Introduction

1.1. Definitions

1.2. Another definition of convergence of ideals 4

1.3. Main results 5

1.4. Examples 6

1.5. Open questions

1.6. Acknowledgements \& origins of the question 8

2. Convergence of ideals 8

3. The Douady space and the cycle space of $\Omega \quad 10$

Key words and phrases. pluricomplex Green function, complex Monge-Ampère equation, residues, analytic disks, ideals of holomorphic functions, Hilbert-Samuel multiplicity, Briançon-Skoda theorem. 2010 Mathematics Subject Classification 32U35, 32A27.

Part of this work was carried out during the stays of the last-named author in the University of Iceland, and of the third-named author in the Université Paul Sabatier, Toulouse, made possible by two Partenariats Hubert Curien "Jules Verne" in 2006-2007 (12339SE) and in 2008-2009 (18980ZB), financed by the French Ministère des Affaires Etrangères and the Icelandic Ministry of Education, Science and Culture. Another part was done during the stay of the second-named author in the Université Paul Sabatier as an invited professor in June 2009. 
3.1. The Douady space of $\Omega \quad 10$

3.2. The cycle space of $\Omega \quad 11$

3.3. Topology of the Douady-space of $\Omega \quad 12$

4. General inequalities $\quad 13$

4.1. First estimates $\quad 13$

4.2. Inequalities involving limit ideals $\quad 15$

4.3. Uniform convergence 17

5. Convergence and Non-Convergence $\quad 18$

5.1. Proof of Theorem 1.8 18

5.2. Proof of Theorem 1.11 20

6. Example : two and three points systems 21

6.1. The case of two points 21

6.2. When all directions coincide $\quad 22$

6.3. Three points, two directions : limit ideal 22

6.4. First reduction : changing the axes 23

6.5. Simplified model cases 27

6.6. Lower estimate 28

6.7. Upper estimates for the generic case 29

6.8. Upper estimates for the degenerate case

References $\quad 39$

\section{INTRODUCTION}

1.1. Definitions. The aim of this paper is to study convergence of pluricomplex Green functions with simple logarithmic poles at finitely many points as the poles tend to a single point. Pluricomplex Green functions with logarithmic singularities have been studied by many authors at different levels of generality. See e.g. Demailly [10], [29], Lempert [19], Lelong [18], Lárusson and Sigurdsson [17], and Rashkovskii and Sigurdsson [24]. We restrict our attention to the case when $\Omega$ is a hyperconvex, bounded, contractible domain in $\mathbb{C}^{n}$ containing the origin 0 and we let $\mathcal{O}(\Omega)$ denote the space of all holomorphic functions on $\Omega$. If $\mathcal{I}$ is an ideal in $\mathcal{O}(\Omega)$, then we let $V(\mathcal{I})$ denote the zero variety of $\mathcal{I}$, consisting of all common zeros of the functions in $\mathcal{I}$, and for every subset $S$ of $\Omega$ we let $\mathcal{I}(S)$ denote the ideal of all functions vanishing on $S$.

We will only consider ideals $\mathcal{I}$ in $\mathcal{O}(\Omega)$, such that $V(\mathcal{I})$ is a finite set. The elements of $\mathcal{I}$ may be defined by local conditions, but by Cartan's Theorem B, there are finitely many generators $\psi_{j} \in \mathcal{O}(\Omega)$ such that for any $f \in \mathcal{I}$, there exists $h_{j} \in \mathcal{O}(\Omega)$ such that $f=\sum_{j} h_{j} \psi_{j}$, see e.g. [16, Theorem 7.2.9, p. 190]. 
Definition 1.1. Let $\mathcal{I}$ be an ideal of $\Omega$. For each $a \in \Omega$, let $\left(\psi_{a, i}\right)_{i}$ be a (local) system of generators. Then

$$
\begin{aligned}
G_{\mathcal{I}}^{\Omega}(z):=\sup \left\{u(z): u \in P S H_{-}(\Omega),\right. \\
\\
\left.u(z) \leq \max _{i} \log \left|\psi_{a, i}\right|+O(1), \forall a \in \Omega\right\} .
\end{aligned}
$$

Note that the condition is meaningful only when $a \in V(\mathcal{I})$. It can be proved that $\mathcal{I} \subset \mathcal{J}$ implies $G_{\mathcal{I}} \leq G_{\mathcal{J}}$. In the special case when $S$ is a finite set in $\Omega$ and $\mathcal{I}=\mathcal{I}(S)$, we write $G_{\mathcal{I}(S)}=G_{S}$ : this case reduces to Pluricomplex Green functions with logarithmic singularities.

We know that $G_{S}$ depends continuously on the poles when those remain a fixed distance apart [18], and would like to know what happens when $S$ coalesces to a single point.

The setup of our problem is the following. Let $A$ be a subset of $\mathbb{C}$ with the origin in its closure $\bar{A}$ and let $\left(\mathcal{I}_{\varepsilon}\right)_{\varepsilon \in A}$ be a family of ideals in $\mathcal{O}(\Omega)$. We are interested in the convergence of $G_{\mathcal{I}_{\varepsilon}}$ as $A \ni \varepsilon \rightarrow 0$ in general and in particular for the special case when $\mathcal{I}_{\varepsilon}=\mathcal{I}\left(S_{\varepsilon}\right)$, where $S_{\varepsilon}$ is a set of $N$ distinct points $a_{1}^{\varepsilon}, \ldots, a_{N}^{\varepsilon}$ all tending to 0 .

We consider ideals of holomorphic functions as points in the Douady space, with the attendant topology (see Section 3 for definitions).

When convergence is locally uniform, one inequality always holds between the limit Green function and the one derived from limits of ideals.

Proposition 1.2. If $G_{\mathcal{I}_{\varepsilon}}$ converges uniformly to $g$ on compact subsets of $\Omega \backslash\{0\}$, and if the family $\left(\mathcal{I}_{\varepsilon}\right)$ converges, then

$$
G_{\varepsilon \rightarrow 0} \operatorname{Iim}_{\varepsilon} \leq \lim _{\varepsilon \rightarrow 0} G_{\mathcal{I}_{\varepsilon}}
$$

This is proved in Section 4, after Lemma 4.5.

The one-pole pluricomplex Green function can be seen as a fundamental solution to the complex Monge-Ampère operator $\left(d d^{c}\right)^{n}$, where $d=\partial+\bar{\partial}, d^{c}:=\frac{1}{2 \pi i}(\partial-\bar{\partial})$. The choice of constant in $d^{c}$ ensures that $\left(d d^{c} \log \|\cdot\|\right)^{n}=\delta_{0}$, the Dirac mass at the origin.

When $f \in \mathcal{C}^{2},\left(d d^{c} f\right)^{n}$ is a multiple of the determinant of the complex Hessian matrix. This operator can be extended to plurisubharmonic functions which are bounded outside of a compact subset of $\Omega$ [2], [11, Chap. III, §3]. If $u$ is plurisubharmonic and $\left(d d^{c} u\right)^{n}=0$ on an open set $\omega$, we say that $u$ is maximal plurisubharmonic on $\omega$. This is equivalent to a form of the maximum principle: if $\omega_{0} \Subset \omega, v \in P S H\left(\omega_{0}\right)$ and $u \geq v$ on $\partial \omega_{0}$, then $u \geq v$ on $\omega_{0}$. 
Corollary 1.3. Under the hypotheses of Proposition 1.2,

$$
\left(d d^{c} \lim _{\varepsilon \rightarrow 0} G_{\mathcal{I}_{\varepsilon}}\right)^{n} \leq\left(d d^{c} G_{\lim _{\varepsilon \rightarrow 0} \mathcal{I}_{\varepsilon}}\right)^{n} .
$$

In particular, since $V\left(\lim _{\varepsilon \rightarrow 0} \mathcal{I}_{\varepsilon}\right)=\{0\}$, then $\lim _{\varepsilon \rightarrow 0} G_{\mathcal{I}_{\varepsilon}}$ is maximal plurisubharmonic outside the origin.

This is proved at the end of Section 4 .

1.2. Another definition of convergence of ideals. Since many analysts, including some authors of the present work, are not familiar with the Douady space, we have found it useful to work with a more concrete approach to the convergence of ideals. It also allows us to work with notions of limes superior and inferior when the family of ideals fails to converge.

Definition 1.4. We call lower limit of $\left(\mathcal{I}_{\varepsilon}\right)_{\varepsilon \in A}$, denoted by $\liminf _{A \ni \varepsilon \rightarrow 0} \mathcal{I}_{\varepsilon}$, the set of all $f \in \mathcal{O}(\Omega)$ such that $f_{\varepsilon} \rightarrow f$ locally uniformly, i.e. uniformly on every compact subset of $\Omega$, as $A \ni \varepsilon \rightarrow 0$, where $f_{\varepsilon} \in \mathcal{I}_{\varepsilon}$.

We call upper limit of $\left(\mathcal{I}_{\varepsilon}\right)_{\varepsilon \in A}$, denoted by $\lim \sup \mathcal{I}_{\varepsilon}$, the subspace of $\mathcal{O}(\Omega)$ generated by all functions $f$ such that $f_{j} \rightarrow f$ locally uniformly, as $j \rightarrow \infty$ for some sequence $\varepsilon_{j} \rightarrow 0$ in $A$ and $f_{j} \in \mathcal{I}_{\varepsilon_{j}}$.

If they are equal then we say that the family $\mathcal{I}_{\varepsilon}$ converges and write $\lim _{A \ni \varepsilon \rightarrow 0} \mathcal{I}_{\varepsilon}$ for the common value of the upper and lower limits.

Of course $\liminf _{A \ni \varepsilon \rightarrow 0} \mathcal{I}_{\varepsilon} \subset \limsup _{A \ni \varepsilon \rightarrow 0} \mathcal{I}_{\varepsilon}$, and it is easy to see that the lower and upper limits of $\left(\mathcal{I}_{\varepsilon}\right)_{\varepsilon \in A}$ are ideals in $\mathcal{O}(\Omega)$. If it is clear from the context which set $A$ we are referring to, then we drop the symbol $A \ni$ from the subscript. Properties following from this definition are given in Section 2 .

This is equivalent to the notion of convergence inherited from the Douady space, see Proposition 3.1 and the Remark following it, in Section 3

The inequality always between the limit Green function and the Green function of a limit ideal survives under much weaker hypotheses.

Proposition 1.5. If $V\left(\liminf _{\varepsilon \rightarrow 0} \mathcal{I}_{\varepsilon}\right)=\{0\}$, then

$$
G_{\liminf _{\varepsilon \rightarrow 0}} \mathcal{I}_{\varepsilon} \leq \liminf _{\varepsilon \rightarrow 0} G_{\mathcal{I}_{\varepsilon}}+O(1) .
$$

This Proposition will be proved in Section 4, after Lemma 4.2.

Still without convergence of ideals, but under more stringent convergence hypotheses for the Green functions, we have the following. 
Proposition 1.6. If $G_{\mathcal{I}_{\varepsilon}}$ converges uniformly to $g$ on compact subsets of $\Omega \backslash\{0\}$, then $G_{\limsup _{\varepsilon \rightarrow 0} \mathcal{I}_{\varepsilon}} \leq g$.

This is proved in Section 4, after Lemma 4.5.

1.3. Main results. In some privileged situations, equality will hold in Proposition 1.2.

Definition 1.7. The family of ideals $\left(\mathcal{I}_{\varepsilon}\right)$ satisfies the Uniform Complete Intersection Condition if for any $\varepsilon$, there exists a map $\Psi_{0}$ and maps $\Psi_{\varepsilon}$ from a neighborhood of $\bar{\Omega}$ to $\mathbb{C}^{n}$ such that $\Psi_{0}$ is proper from $\Omega$ to $\Psi_{0}(\Omega)$, and

(1) $\left\{a_{j}^{\varepsilon}, 1 \leq j \leq N\right\}=\Psi_{\varepsilon}^{-1}\{0\}$, for all $\varepsilon$;

(2) For all $\varepsilon \neq 0,1 \leq j \leq N$ and $z$ in a neighborhood of $a_{j}^{\varepsilon}$,

$$
\left|\log \left\|\Psi_{\varepsilon}(z)\right\|-\log \left\|z-a_{j}^{\varepsilon}\right\|\right| \leq C(\varepsilon)<\infty ;
$$

(3) $\lim _{\varepsilon \rightarrow 0} \Psi_{\varepsilon}=\Psi_{0}=\left(\Psi_{0}^{1}, \ldots, \Psi_{0}^{n}\right)$, uniformly on $\bar{\Omega}$.

Notice that the first two conditions imply $\mathcal{I}_{\varepsilon}=\left\langle\Psi_{\varepsilon}^{1}, \ldots, \Psi_{\varepsilon}^{n}\right\rangle$.

Theorem 1.8. Let $\left(\mathcal{I}_{\varepsilon}\right)$ be a family of ideals satifying the complete intersection condition, set $S_{\varepsilon}=V\left(\mathcal{I}_{\varepsilon}\right)$ and $\mathcal{I}_{0}=\left\langle\Psi_{0}^{1}, \ldots, \Psi_{0}^{n}\right\rangle$. Then

(1) $\lim _{\varepsilon \rightarrow 0} \mathcal{I}_{\varepsilon}=\mathcal{I}_{0}$,

(2) $\lim _{\varepsilon \rightarrow 0} G_{\varepsilon}=G_{\mathcal{I}_{0}}$, and the convergence is locally uniform on $\Omega \backslash\{0\}$.

This theorem is proved in Section 5 .

We shall see that the above equalities only rarely hold. We need to recall the notions of length and multiplicity for an ideal.

Definition 1.9. Let $\mathcal{I}$ be an ideal of $\mathcal{O}(\Omega)$ such that $V(\mathcal{I})$ is a finite set.

(1) The length of $\mathcal{I}$ is $\ell(\mathcal{I}):=\operatorname{dim} \mathcal{O} / \mathcal{I}$.

(2) The Hilbert-Samuel multiplicity of $\mathcal{I}$ is

$$
e(\mathcal{I})=\lim _{k \rightarrow \infty} \frac{n !}{k^{n}} \ell\left(\mathcal{I}^{k}\right)
$$

(3) If $\mathcal{I}$ admits a set of $n$ generators, it is called a parameter ideal, or complete intersection ideal.

Multiplicity is no smaller than length, and they match only in very particular cases.

Proposition 1.10. [30, Ch. VIII, Theorem 23] If $V(\mathcal{I})=\{a\}, e(\mathcal{I}) \geq$ $\ell(\mathcal{I})$, and $e(\mathcal{I})=\ell(\mathcal{I})$ if and only if $\mathcal{I}$ is a complete intersection ideal. 
The next theorem is our main general result. Its converse direction shows that the second conclusion of Theorem 1.8 can be deduced from hypotheses about the limit ideal only.

Theorem 1.11. Let $\mathcal{I}_{\varepsilon}=\mathcal{I}\left(S_{\varepsilon}\right)$, where $S_{\varepsilon}$ is a set of $N$ points all tending to 0 and assume that $\lim _{\varepsilon \rightarrow 0} \mathcal{I}_{\varepsilon}=\mathcal{I}$. Then $\left(G_{\mathcal{I}_{\varepsilon}}\right)$ converges to $G_{\mathcal{I}}$ locally uniformly on $\Omega \backslash\{0\}$ if and only if $\mathcal{I}$ is a complete intersection ideal.

This theorem is proved in Section 5 .

1.4. Examples. Note that in the case where $\mathcal{I}$ requires more than $n$ generators, the sequence $\left(G_{\mathcal{I}_{\varepsilon}}\right)$ may converge, to a limit which is not the Green function of an ideal. The first case when this can occur is when $n=2$ and $N=3$.

We fix some notations. As usual, for $a \in \Omega$,

$$
\mathfrak{M}_{a}:=\mathcal{I}_{\{a\}}=\left\langle z_{1}-a_{1}, \ldots, z_{n}-a_{n}\right\rangle \text {. }
$$

Recall that

$$
\ell\left(\mathfrak{M}_{a}\right)=1, \quad \ell\left(\mathfrak{M}_{a}^{p}\right)=\left(\begin{array}{c}
n+p-1 \\
n
\end{array}\right), p \geq 1 .
$$

If $\mathcal{I}$ is the ideal of all functions vanishing at $N$ distinct points $a_{1}, \ldots, a_{N}$, then $\ell(\mathcal{I})=N$.

For $\varepsilon \in \mathbb{C}$, let $a_{i}^{\varepsilon} \in \Omega \subset \mathbb{C}^{2}, 1 \leq i \leq 3$, be three distinct points, $S_{\varepsilon}:=$ $\left\{a_{1}^{\varepsilon}, a_{2}^{\varepsilon}, a_{3}^{\varepsilon}\right\}$, with $\lim _{\varepsilon \rightarrow 0} a_{i}^{\varepsilon}=0,1 \leq i \leq 3 ; G_{\varepsilon}:=G_{S_{\varepsilon}}, \mathcal{I}_{\varepsilon}:=\mathcal{I}\left(S_{\varepsilon}\right)$.

Let $v_{i}^{\varepsilon}:=\left[a_{j}^{\varepsilon}-a_{k}^{\varepsilon}\right] \in \mathbb{P}^{1}$, where $\{i, j, k\}=\{1,2,3\}, j, k \neq i$.

Theorem 1.12. Assume that there exists $A \subset \mathbb{C} \backslash\{0\}$ with $0 \in \bar{A}$ such that $v_{i}=\lim _{A \ni \varepsilon \rightarrow 0} v_{i}^{\varepsilon}$. Let $\tilde{v}_{i} \in \mathbb{C}^{2}, 1 \leq i \leq 3$, be such that $\left\|\tilde{v}_{i}\right\|=1$ and $\left[\tilde{v}_{i}\right]=v_{i}$.

(i) If there exist $i, j$ such that $v_{i} \neq v_{j}$, then $\lim _{\varepsilon \rightarrow 0} \mathcal{I}_{\varepsilon}=\mathfrak{M}_{0}^{2}$, and $G_{\varepsilon} \rightarrow g$ locally uniformly on $\Omega \backslash\{0\}$ as $A \ni \varepsilon \rightarrow 0$, where $g \in P S H(\Omega)$ is extremal on $\Omega \backslash\{0\}, g(z) \leq \frac{3}{2} \log \|z\|+O(1)$ as $z \rightarrow 0$, and for $\zeta \in \mathbb{C}$ we have

$$
g(\zeta v)= \begin{cases}2 \log |\zeta|+O(1), & v=\tilde{v}_{i}, \quad 1 \leq i \leq 3 \\ \frac{3}{2} \log |\zeta|+O(1), & v \in \mathbb{C}^{2} \backslash \cup_{1 \leq i \leq 3} \mathbb{C} \tilde{v}_{i} .\end{cases}
$$

(ii) There exists an infinite set $\mathfrak{A}$ of families $a:=\left\{\left\{a_{1}^{\varepsilon}, a_{2}^{\varepsilon}, a_{3}^{\varepsilon}\right\}, \varepsilon \in \mathbb{C}\right\}$, such that for any $a \in \mathfrak{A}, v_{1}=v_{2}=v_{3}=[1: 0], \lim _{\varepsilon \rightarrow 0} \mathcal{I}_{\varepsilon}=\mathcal{I}^{a}$ and $\lim _{\varepsilon \rightarrow 0} G_{\varepsilon}=g^{a}$, where the ideals $\mathcal{I}^{a}$ (respectively the functions $g^{a}$ ) are distinct for distinct values of $a$. 
This theorem is proved in Section 6 .

Remark 1. In Section 6, we also give a general picture of the convergence (or not) of Green functions for two poles tending to the origin, and in the case of three poles, after Proposition 6.2, more detailed descriptions of the possible functions $g$, which depend on whether the $v_{i}$ are all distinct or not.

Remark 2. Since $\mathbb{P}^{1}$ is compact, any family $\left\{v_{i}^{\varepsilon}\right\}_{\varepsilon \in E}$ admits a convergent subfamily. For a $E^{\prime} \subset E$ such that each $\left\{v_{i}^{\varepsilon}\right\}_{\varepsilon \in E^{\prime}}$ is convergent, one of the above cases will apply. If different subfamilies lead to distinct limits, the original family $\left\{G_{\varepsilon}, \varepsilon \in E\right\}$ is not convergent. In particular, if $\mathcal{V}$ denotes the cluster set of the sets $\left\{v_{i}^{\varepsilon}, 1 \leq i \leq 3\right\}$, and if we have $\# \mathcal{V} \geq 4$, the family $\left\{G_{\varepsilon}, \varepsilon \in E\right\}$ is not convergent.

1.5. Open questions. When dealing with plurisubharmonic functions, it is natural to consider "weak" convergence, in the $L_{\text {loc }}^{1}$ sense. This is in fact enough to obtain first estimates of the mass of a limit of Green functions.

Proposition 1.13. Let $S_{\varepsilon}$ be a set of $N$ points all tending to 0. Suppose that $g=\lim _{\varepsilon \rightarrow 0} G_{S_{\varepsilon}}$ exists in $L_{l o c}^{1}$. Then

$$
\left(d d^{c} g\right)^{n}(\Omega) \leq \liminf _{\varepsilon \rightarrow 0}\left(d d^{c} G_{S_{\varepsilon}}\right)^{n}(\Omega)=N .
$$

The proof of this useful fact, which relies mostly on a result in [6], will be given in Section 4, before Lemma 4.2.

However, to obtain a better behavior we would like to make sure, in particular, that the Monge-Ampère mass of the limit function is concentrated at the origin. This happens when convergence is locally uniform on $\Omega \backslash\{0\}$ (Corollary 1.3). Given the rigid nature of Green functions, is it possible to find any situation where this better kind of convergence is not realized?

Theorem 1.12 and the following remarks show that the same ideal $\mathfrak{M}_{0}^{2}$ can be obtained as limit of many distinct families $\mathcal{I}_{\varepsilon}$ of ideals based on three points, which give rise to distinct families of Green functions with different limits $g$, all of which, however, share the property that $\left(d d^{c} g\right)^{2}=3 \delta_{0}=\ell\left(\mathfrak{M}_{0}^{2}\right) \delta_{V\left(\mathfrak{M}_{0}^{2}\right)}$.

Is there any natural way to associate to an ideal $\mathcal{I}$ such that $V(\mathcal{I})=$ $\{0\}$ a plurisubharmonic function $h_{\mathcal{I}}$ such that $\left(d d^{c} h_{\mathcal{I}}\right)^{n}=\ell(\mathcal{I}) \delta_{0}$ ?

Any such correspondence, however, is not likely to be one-to-one ; for instance, if we take any two independent linear forms $\psi_{1}, \psi_{2}$ on $\mathbb{C}^{2}$, then in general $\left\langle\psi_{1}^{2}, \psi_{2}^{2}\right\rangle \neq\left\langle z_{1}^{2}, z_{2}^{2}\right\rangle$; however they have the same Green functions, which by Theorem 1.8 are the limits of Green functions of certain families of 4 -point sets. 
1.6. Acknowledgements \& origins of the question. It is known that the Green function is smaller than another holomorphic invariant, the Lempert function [8], 4]. When looking for cases where this inequality is strict, for single poles, we had to consider situations where $S=S_{\varepsilon}$ depended on a parameter, and the poles of $S$ were tending to a same point as $\varepsilon \rightarrow 0$ [26]. This led us to consider $\lim _{\varepsilon \rightarrow 0} G_{S_{\varepsilon}}$.

The work on this paper spanned many years, from the first time that Nguyen Van Trao asked the last-named author about the limit of a Green function with three poles, to the present. Over that period, we have benefitted from many conversations with kind and patient colleagues about one aspect or another of this work. Although writing down a list runs the risk of omission, we'd like to thank (from A to Z) Eric Amar, Mats Andersson, Eric Bedford, Jean-Paul Calvi, Urban Cegrell, Jean-Pierre Demailly, Philippe Eyssidieux, Vincent Guedj, Nguyen Quang Dieu, Mikael Passare, Evgeny Poletsky, Mark Spivakovsky, Elizabeth Wulcan, Alain Yger, and Ahmed Zeriahi.

\section{Convergence of ideals}

Lemma 2.1. If $\ell\left(\mathcal{I}_{\varepsilon}\right) \leq N$ for all $\varepsilon \in A$, then $\ell\left(\limsup _{A \ni \varepsilon \rightarrow 0} \mathcal{I}_{\varepsilon}\right) \leq N$.

Proof. Suppose $k>N$ and $f^{1}, \ldots, f^{k} \in \mathcal{O}(\Omega)$. By the hypothesis, for any $\varepsilon \in A$, there exist $\lambda_{\varepsilon}^{m} \in \mathbb{C}, 1 \leq m \leq k$, not all zero, such that

$$
\sum_{m=1}^{k} \lambda_{\varepsilon}^{m} f^{m}=g_{\varepsilon} \in \mathcal{I}_{\varepsilon} .
$$

We can normalize the coefficients $\lambda_{\varepsilon}^{m}$ such that $\max _{m}\left|\lambda_{\varepsilon}^{m}\right|=1$, and by passing to a subsequence, and renumbering the $f^{m}$ if needed, we can assume $\lambda_{\varepsilon}^{1}=1,\left|\lambda_{\varepsilon}^{m}\right| \leq 1$ for $2 \leq m \leq k$. Then by using the compactness of $\overline{\mathbb{D}}$, we can pass to another subsequence $\varepsilon_{j}$ such that $\lim _{j \rightarrow \infty} \lambda_{\varepsilon_{j}}^{m}=\lambda^{m} \in \overline{\mathbb{D}}, 2 \leq m \leq k$. This implies that $\lim g_{\varepsilon_{j}}=: g$ exists (locally uniformly) and

$$
f^{1}+\sum_{m=2}^{k} \lambda^{m} f^{m}=g \in \limsup _{A \ni \varepsilon \rightarrow 0} \mathcal{I}_{\varepsilon} .
$$

So the system $\left[f^{1}\right], \ldots,\left[f^{k}\right]$ is not linearly independent in $\mathcal{O}(\Omega) / \lim \sup \mathcal{I}_{\varepsilon}$.

Lemma 2.2. If for each $\varepsilon \in A, \mathcal{I}_{\varepsilon}$ is an ideal based on $N$ distinct points, all tending to 0 as $\varepsilon$ tends to 0 , and if $\ell\left(\mathcal{I}_{\varepsilon}\right) \geq N$ for all $\varepsilon \in A$, then $\ell\left(\liminf _{A \ni \varepsilon \rightarrow 0} \mathcal{I}_{\varepsilon}\right) \geq N$. 
Proof. Let $\pi_{j}$ denote the projection to the $j$-th coordinate axis. Let $V\left(\mathcal{I}_{\varepsilon}\right)=\left\{a_{1}^{\varepsilon}, \ldots, a_{N}^{\varepsilon}\right\}$.

We can split $A$ into a finite union of sets $A_{k}$ such that for each $k$, $j \in\{1, \ldots, n\}, \# \pi_{j}\left(\left\{a_{1}^{\varepsilon}, \ldots, a_{N}^{\varepsilon}\right\}\right)=N_{k, j}$ is independent of $\varepsilon$. The sets $A_{k}$ which don't have the origin in their closure do not concern us; let us now consider one that does, which we will denote again by $A$, and write $N_{j}=N_{k, j}$. Then let

$$
P_{\varepsilon}:=\pi_{1}\left(\left\{a_{1}^{\varepsilon}, \ldots, a_{N}^{\varepsilon}\right\}\right) \times \cdots \times \pi_{n}\left(\left\{a_{1}^{\varepsilon}, \ldots, a_{N}^{\varepsilon}\right\}\right) \text { (cartesian product) }
$$

and $\mathcal{J}_{\varepsilon}$ the set of all $f \in \mathcal{O}(\Omega)$ vanishing on $P_{\varepsilon}$. It is easy to see that $\ell\left(\mathcal{J}_{\varepsilon}\right)=\# P_{\varepsilon}=\prod_{j=1}^{n} N_{j} \leq N^{n}$.

Lemma 2.3. Under the hypotheses of Lemma 2.2,

$\lim _{\varepsilon \rightarrow 0} \mathcal{J}_{\varepsilon}=\mathcal{J}:=\left\{f \in \mathcal{O}(\Omega): \frac{\partial^{k_{1}+\cdots+k_{n}} f}{\partial z_{1}^{k_{1}} \ldots \partial z_{n}^{k_{n}}}(0)=0,1 \leq j \leq n, 0 \leq k_{j} \leq N_{j}-1\right\}$.

Lemma 2.3 will be proved below. Note that it is a special case of the first statement in Theorem 1.8,

Let $\mathcal{I}:=\liminf _{A \ni \varepsilon \rightarrow 0} \mathcal{I}_{\varepsilon}$. Since $\mathcal{J}_{\varepsilon} \subset \mathcal{I}_{\varepsilon}$, it is easy to see that $\mathcal{J} \subset \mathcal{I}$.

Then $\ell(\mathcal{I})=\ell(\mathcal{J})-\operatorname{dim}(\mathcal{I} / \mathcal{J})=\prod_{j=1}^{n} N_{j}-\operatorname{dim}(\mathcal{I} / \mathcal{J})$, and

$$
N \leq \ell\left(\mathcal{I}_{\varepsilon}\right)=\ell\left(\mathcal{J}_{\varepsilon}\right)-\operatorname{dim}\left(\mathcal{I}_{\varepsilon} / \mathcal{J}_{\varepsilon}\right)=\prod_{j=1}^{n} N_{j}-\operatorname{dim}\left(\mathcal{I}_{\varepsilon} / \mathcal{J}_{\varepsilon}\right)
$$

So to prove Lemma 2.2, it will be enough to show that $\operatorname{dim}(\mathcal{I} / \mathcal{J}) \leq$ $\prod_{j=1}^{n} N_{j}-N$, by using the fact that $\operatorname{dim}\left(\mathcal{I}_{\varepsilon} / \mathcal{J}_{\varepsilon}\right) \leq \prod_{j=1}^{n} N_{j}-N$.

Let $k>\prod_{j=1}^{n} N_{j}-N$ and $f^{1}, \ldots, f^{k} \in \mathcal{I}$. By the definition of lim inf, for any $\varepsilon \in A$, there exist $f_{\varepsilon}^{1}, \ldots, f_{\varepsilon}^{k} \in \mathcal{I}_{\varepsilon}$ such that $\lim _{\varepsilon \rightarrow 0} f_{\varepsilon}^{m}=f^{m}$, $1 \leq m \leq k$. By the bound on the dimension of $\mathcal{I}_{\varepsilon} / \mathcal{J}_{\varepsilon}$, there exists $g_{\varepsilon} \in \mathcal{J}_{\varepsilon}$ and coefficients $\lambda_{\varepsilon}^{m} \in \mathbb{C}, 1 \leq m \leq k$, not all zero, such that

$$
\sum_{m=1}^{k} \lambda_{\varepsilon}^{m} f_{\varepsilon}^{m}=g_{\varepsilon}
$$

Now we pass to a subsequence exactly as in the proof of Lemma 2.1, using in addition the convergence of the $f_{\varepsilon}^{m}$, to find

$$
f^{1}+\sum_{m=2}^{k} \lambda^{m} f^{m}=g
$$

with $g=\lim g_{\varepsilon_{j}} \in \limsup _{A \ni \varepsilon \rightarrow 0} \mathcal{J}_{\varepsilon}=\mathcal{J}$, since the family $\left(\mathcal{J}_{\varepsilon}\right)$ converges. This proves that the system $\left[f^{1}\right], \ldots,\left[f^{k}\right]$ is not linearly independent in $\mathcal{I} / \mathcal{J}$. 
Corollary 2.4. If $\left(\mathcal{I}_{\varepsilon}\right)$ is a convergent family of point-based ideals, $\ell\left(\lim _{\varepsilon \rightarrow 0} \mathcal{I}_{\varepsilon}\right)=\lim _{\varepsilon \rightarrow 0} \ell\left(\mathcal{I}_{\varepsilon}\right)$.

Proof of Lemma 2.3. Denote the elements of $\pi_{j}\left(\left\{a_{1}^{\varepsilon}, \ldots, a_{N}^{\varepsilon}\right\}\right)$ by $\alpha_{j}^{i, \varepsilon}$, $1 \leq i \leq N_{j}, 1 \leq j \leq n$. Let $\psi_{j}^{\varepsilon}(\zeta):=\left(\zeta-\alpha_{j}^{1, \varepsilon}\right) \cdots\left(\zeta-\alpha_{j}^{N_{j}, \varepsilon}\right)$, for $\zeta \in \mathbb{C}$.

Suppose that $f \in \mathcal{J}$. Then there are holomorphic functions $h_{j}$, $1 \leq j \leq n$, such that

$$
f(z)=\sum_{j=1}^{n} z_{j}^{N_{j}} h_{j}(z)
$$

Setting

$$
f_{\varepsilon}(z)=\sum_{j=1}^{n} \psi_{j}^{\varepsilon}\left(z_{j}\right) h_{j}(z)
$$

we have a family of $f_{\varepsilon} \in \mathcal{J}_{\varepsilon}$ such that $f=\lim _{\varepsilon \rightarrow 0} f_{\varepsilon}$.

Conversely, let $\lim _{k \rightarrow \infty} f_{\varepsilon_{k}}=f$, where $f_{\varepsilon_{k}} \in \mathcal{J}_{\varepsilon_{k}}, \varepsilon_{k} \rightarrow 0$. By rescaling, we might assume that $\overline{\mathbb{D}}^{n} \subset \Omega$. One can prove by induction on $n$ that if $f_{\varepsilon} \in \mathcal{J}_{\varepsilon}$, and $|\varepsilon|$ is small enough so that $\left|\alpha_{j}^{i, \varepsilon}\right|<1$ for all $i, j$, then

$$
\int_{(\partial \mathbb{D})^{n}} \frac{f_{\varepsilon}\left(z_{1}, \ldots, z_{n}\right)}{\prod_{j=1}^{n} \psi_{j}^{\varepsilon}\left(z_{j}\right)} d z_{1} \ldots d z_{n}=0
$$

Applying this to $f_{\varepsilon_{k}}$, and passing to the limit as $k \rightarrow \infty$, we find

$$
\frac{\partial^{N_{1}+\cdots+N_{n}-n} f}{\partial z_{1}^{N_{1}-1} \ldots \partial z_{n}^{N_{n}-1}}(0)=\frac{1}{(2 \pi i)^{n}} \int_{(\partial \mathbb{D})^{n}} \frac{f\left(z_{1}, \ldots, z_{n}\right)}{\prod_{j=1}^{n} z_{j}^{N_{j}}} d z_{1} \ldots d z_{n}=0 .
$$

The same result will hold replacing each $N_{j}$ by any $k_{j} \leq N_{j}$, simply by taking appropriate subsets of $P^{\varepsilon}$.

\section{The Douady space and the Cycle Space of $\Omega$}

The aim of this section is to show that the convergence notion we introduced in the previous section is in fact equivalent to convergence in the Douady space of $\Omega$ (see proposition 3.1 below.)

3.1. The Douady space of $\Omega$. A flat and proper family of subspaces of $\Omega$ is a pair of complex spaces $(S, Z)$ such that $Z$ is a subspace of $S \times \Omega$ and such that the natural projection $\pi: Z \rightarrow S$ is a flat and proper holomorphic map. The space $S$ is called the parameter space and the space $Z$ is called the graph of the family.

Due to the natural identification $\{s\} \times \Omega \simeq \Omega$ we think of this as a family of compact subspaces $\left(Z_{s}\right)_{s \in S}$ in $\Omega$ parametrized by the space $S$ where $Z_{s}$ denotes the analytic fibre of $\pi$ over the point $s$. 
Since $\pi$ is a proper holomorphic map the spaces $Z_{s}$ are all compact complex subspaces of $\Omega$ and consequently finite. Hence $\pi$ is a finite (and proper) holomorphic map and in that case one knows that $\pi$ is flat if and only if the direct image sheaf $\pi_{*} \mathcal{O}_{Z}$ is a locally free $\mathcal{O}_{S}-$ module (of finite rank); see [14]. This again is equivalent to say that, on each connected component of $S$, all the fibres of the sheaf $\pi_{*} \mathcal{O}_{Z}$ are complex vector spaces of the same dimension. But the fibre of $\pi_{*} \mathcal{O}_{Z}$ at the point $s$ is naturally isomorphic to the quotient space $\Gamma\left(\Omega, \mathcal{O}_{\Omega}\right) / I_{s}$ where $I_{s}$ denotes the ideal of $Z_{s}$ in $\Gamma\left(\Omega, \mathcal{O}_{\Omega}\right)$. In other words the map $\pi$ is flat if and only if the length of $I_{s}$ is a locally constant function on $S$.

From [13] we know that every complex space admits a universal flat and proper family. In our setting this means that there exists a flat and proper family $(D, X)$ of subspaces in $\Omega$ having the following universal property:

- If $(S, Z)$ is any flat and proper family of subspaces of $\Omega$ then there exists a unique holomorphic map $f: S \rightarrow D$ such that $Z$ is the pullback of $X$ by $f \times \mathrm{id}_{\Omega}$.

This implies in particular that the space $D$, which is by definition the Douady space of $\Omega$, parametrizes (in a one-to-one way) all the compact subspaces of $\Omega$; in other words every compact subspace of $\Omega$ appears exactly once in the family $\left(X_{t}\right)_{t \in D}$. In the sequel we will denote $\mathcal{I}_{t}$ the $\mathcal{O}_{\Omega}$-ideal corresponding to $X_{t}$ and put $I_{t}:=\Gamma\left(\Omega, \mathcal{I}_{t}\right)$.

We have a natural decomposition $D=\sqcup_{k \geq 1} D_{k}$, where $D_{k}$ denotes the open subspace of $D$ formed by those $t$ such that $I_{t}$ is of length $k$.

3.2. The cycle space of $\Omega$. Let $\operatorname{Sym}^{k}(\Omega)$ denote the $k$-th symmetric product of $\Omega$, i.e. the normal complex space obtained as a quotient of $\Omega^{k}$ by the natural action of the $k$-th symmetric group. One can think of every element in $\mathrm{Sym}^{k}$ as a given set of points each with a multiplicity. These elements are usually called 0-cycles and each one of them can be expressed in a unique way as $n_{1} x_{1}+\cdots+n_{l} x_{l}$, where $x_{1}, \ldots, x_{l}$ are mutually distinct, $n_{j}$ is the multiplicity of $x_{j}$ and consequently $n_{1}+\cdots+n_{l}=k$.

Since every compact complex subspace of $\Omega$ is finite the disjoint union

$$
\mathcal{C}:=\bigsqcup_{k \geq 1} \operatorname{Sym}^{k}(\Omega)
$$

is the cycle space of $\Omega$. 
For every $k$ we have a natural holomorphic map from the reduction of $D_{k}$ to $\operatorname{Sym}^{k}(\Omega)$ defined in the following way (see for instance [1] or [20]):

To each $t$ in $D_{k}$ we associate the 0-cycle $n_{1} x_{1}+\cdots+n_{l} x_{l}$ where $x_{1}, \ldots, x_{l}$ are the mutually distinct points of $X_{t}$ and $n_{j}:=$ $\operatorname{dim}_{\mathbb{C}} \mathcal{O}_{\Omega, x_{j}} /\left(\mathcal{I}_{t}\right)_{x_{j}}$.

Moreover this map is proper [21]. Hence we obtain a proper holomorphic map

$$
\mu: D_{\text {red }} \longrightarrow \mathcal{C}
$$

where $D_{\text {red }}$ denotes the reduction of $D$.

3.3. Topology of the Douady-space of $\Omega$. Since the topological space underlying $D$ is first countable the following proposition characterizes its topology.

Proposition 3.1. Let $\left(t_{j}\right)_{j \geq 1}$ be a sequence in $D$ and let a be a point in $D$. Then the sequence $\left(t_{j}\right)_{j \geq 1}$ converges to $a$ in $D$ if and only if the following two conditions are satisfied:

(1) The sequence $\left(\mu\left(t_{j}\right)\right)_{j \geq 1}$ converges to $\mu(a)$ in $\mathcal{C}$.

(2) $\lim _{j \rightarrow \infty} I_{t_{j}}=I_{a}$, where the limit is taken in the sense of Definition 1.4 .

Proof. Suppose that the sequence $\left(t_{j}\right)_{j \geq 1}$ converges to $a$ in $D$. Then condition (1) is satisfied because the map $\mu$ is continuous.

To prove that condition (2) is fulfilled it is sufficient to show that $\lim _{t \rightarrow a} I_{t}=I_{a}$. Without loss of generality we may replace $D$ by a Stein open (connected) neighbourhood $T$ of $a$ in $D$ and we may assume $T$ is reduced since flatness is preserved by base change. We still denote $X$ the restriction of the graph $X$ to $T$ and let $\mathcal{I}$ be the corresponding $\mathcal{O}_{T \times \Omega}$-ideal.

Let us first prove that $I_{a} \subseteq \liminf _{t \rightarrow a} I_{t}$. To do so take any function $f$ in $I_{a}$. Then since $T \times \Omega$ is Stein there exists a function $g$ in $\Gamma(T \times \Omega, \mathcal{I})$ such that $g(a, z)=f(z)$ for all $z$ in $X_{a}$. Now for every $t$ in $T$ define a function $f_{t}$ on $\Omega$ by setting

$$
f_{t}(z):=g(t, z) \text {. }
$$

Then it is clear that $f_{t} \in I_{t}$ and we obviously have $f_{t} \rightarrow f$ uniformly on every compact set in $\Omega$.

To prove that $\limsup _{t \rightarrow a} I_{t} \subseteq I_{a}$ suppose we have a sequence of points $\left(x_{\nu}\right)_{\nu}$ in $T$ converging to $a$ and for each $\nu$ a function $f_{\nu}$ in $I_{x_{\nu}}$ such that the sequence $\left(f_{\nu}\right)_{\nu}$ converges locally uniformly to a function $f$ in $\Gamma\left(\Omega, \mathcal{O}_{\Omega}\right)$. Now each $f_{\nu}$ defines a global holomorphic 
section $\sigma_{\nu}$ of the locally free sheaf $\pi_{*} \mathcal{O}_{X}$ on $T$ and they converge locally uniformly to the holomorphic global section $\sigma$ defined by $f$ as $\nu \rightarrow \infty$. Now if $\tau$ is a global section of $\pi_{*} \mathcal{O}_{X}$ defined by a global holomorphic function $g$ on $\Omega$ then the "value", $\tau(t)$, of $\tau$ at a point $t$ in $T$ is the image of $g$ in the $\mathbb{C}$-vector space

$$
\left(\pi_{*} \mathcal{O}_{X}\right)_{t} \otimes_{\mathcal{O}_{T, t}} \mathbb{C} \simeq \Gamma\left(X_{t}, \mathcal{O}_{X_{t}}\right) \simeq \Gamma\left(\Omega, \mathcal{O}_{\Omega}\right) / I_{t}
$$

In particular $\tau(t)=0$ is equivalent to $g \in I_{t}$. Since $\sigma_{\nu}\left(t_{\nu}\right)=0$ it follows that $\sigma(a)=0$ and consequently $f \in I_{a}$.

Conversely, suppose that the sequence $\left(t_{j}\right)_{j \geq 1}$ satisfies the two conditions and assume that it does not tend to $a$. Then the point $a$ admits an open neighbourhood $V$ in $D$ outside of which there exists a subsequence $\left(t_{j_{l}}\right)_{l \geq 1}$ of $\left(t_{j}\right)_{j \geq 1}$.

Since the map $\mu: D_{\text {red }} \longrightarrow \mathcal{C}$ is proper we may, without loss of generality, assume that the subsequence $\left(t_{j_{l}}\right)_{l \geq 1}$ converges to a point $b$ in the fibre $\mu^{-1}(\mu(a))$. From what we proved above it then follows that

$$
I_{a}=\lim _{j \rightarrow \infty} I_{t_{j}}=\lim _{l \rightarrow \infty} I_{t_{j_{l}}}=I_{b}
$$

in contradiction to the fact that $t_{j_{l}} \notin V$ for all $l$.

Remark. Assuming the hypothesis of Section 2 let us denote $\iota$ : $A \rightarrow D$ the canonical mapping that associates to each $\epsilon$ in $A$ the compact complex subspace of $\Omega$ defined by $I_{\epsilon}$. If $I_{0}:=\lim _{\epsilon \rightarrow 0} I_{\epsilon}$ exists then $I_{0}$ defines a point in $D$ and we get

$$
\lim _{\epsilon \rightarrow 0} \iota(\epsilon)=\iota(0) \text {. }
$$

\section{General inequalities}

Suppose throughout this section that we are given a family of ideals $\mathcal{I}_{\varepsilon} \subset \mathcal{O}(\Omega)$.

\subsection{First estimates.}

Lemma 4.1. Let $\Omega$ be a bounded hyperconvex domain such that $0 \in \Omega$. Let $S_{\varepsilon}=\left\{a_{j}^{\varepsilon}, 1 \leq j \leq N\right\}$, with $\lim _{\varepsilon \rightarrow 0} a_{j}^{\varepsilon}=0,1 \leq j \leq N$.

Then for any $\delta>0$, there exists $\varepsilon_{0}=\varepsilon_{0}(\delta)>0$ such that for any $z \in \Omega \backslash \bar{B}(0, \delta)$, for any $\varepsilon$ such that $|\varepsilon| \leq \varepsilon_{0}$,

$$
(N+\delta) G_{0}(z) \leq G_{S_{\varepsilon}}(z) \leq(1-\delta) G_{0}(z) .
$$

Remark. This implies that the family $G_{S_{\varepsilon}}$ is equicontinuous near $\partial \Omega$. As a consequence, a subsequence $G_{S_{\varepsilon_{j}}}$ converges uniformly on compacta of $\bar{\Omega} \backslash\{0\}$ if and only if it converges uniformly on compacta of $\Omega \backslash\{0\}$. 
Proof. It is well known that

$$
\sum_{j=1}^{N} G_{a_{j}^{\varepsilon}}(z) \leq G_{S_{\varepsilon}}(z) \leq \min _{1 \leq j \leq N} G_{a_{j}^{\varepsilon}}(z) .
$$

We will compare each of the $G_{a_{j}^{\varepsilon}}(z)$ to $G_{0}(z)$. There are all equal to 0 on $\partial \Omega$.

There are numbers $0<r_{1}<r_{2}, r_{2} \geq 1$, such that $\bar{B}\left(0, r_{1}\right) \subset \Omega \subset$ $B\left(0, r_{2}\right)$, so

$$
\log \frac{\|z\|}{r_{2}} \leq G_{0}(z) \leq \log \frac{\|z\|}{r_{1}} .
$$

Now we take $z$ such that $\|z\|=\delta_{1} \leq \delta, \delta_{1}$ to be chosen below. Then for $\left|a_{j}^{\varepsilon}\right|<\delta$,

$$
G_{a_{j}^{\varepsilon}}(z) \leq \log \frac{\left\|z-a_{j}^{\varepsilon}\right\|}{r_{1}-\left\|a_{j}^{\varepsilon}\right\|} \leq \log \frac{\delta_{1}}{r_{1}}+1,
$$

for $|\varepsilon|$ small enough (depending on $\delta_{1}$ ). We can choose $\delta_{1}$ so small that

$$
\log \delta_{1}-\log r_{1}+1 \leq(1-\delta) \log \delta_{1}-\log r_{2} \leq(1-\delta) \log \frac{\delta_{1}}{r_{2}},
$$

so that $G_{a_{j}^{\varepsilon}}(z) \leq(1-\delta) G_{0}(z)$ when $\|z\|=\delta_{1}$. Since $G_{0}$ is maximal plurisubharmonic on $\Omega \backslash \bar{B}\left(0, \delta_{1}\right)$, and $G_{a_{j}^{\varepsilon}}(z)=(1-\delta) G_{0}(z)=0$ when $z \in \partial \Omega$, the inequality holds on the whole of $\Omega \backslash \bar{B}\left(0, \delta_{1}\right)$.

In a similar way, for $\|z\|=\delta_{1} \leq \delta$, and $\left|a_{j}^{\varepsilon}\right|<\delta$,

$$
G_{a_{j}^{\varepsilon}}(z) \geq \log \frac{\left\|z-a_{j}^{\varepsilon}\right\|}{r_{2}+\left\|a_{j}^{\varepsilon}\right\|} \geq \log \frac{\delta_{1}}{r_{2}}-1,
$$

for $|\varepsilon|$ small enough. We can choose $\delta_{1}$ so small that

$$
\log \delta_{1}-\log r_{2}-1 \geq(1+\delta / N) \log \frac{\delta_{1}}{r_{1}},
$$

so that $G_{a_{j}^{\varepsilon}}(z) \geq(1+\delta / N) G_{0}(z)$ when $\|z\|=\delta_{1}$, and the inequality on the whole of $\Omega \backslash \bar{B}\left(0, \delta_{1}\right)$ follows by maximality of $G_{a_{j}^{\varepsilon}}$.

Proof of Proposition 1.13. The first claim in [6, Lemma 2.1] states that if $u, u_{j} \in \mathcal{F}(\Omega)$ and $u_{j}$ converges weakly to $u$, then for any $w \in$ $P S H_{-}(\Omega)$,

$$
\limsup _{j \rightarrow \infty} \int_{\Omega} w\left(d d^{c} u_{j}\right)^{n} \leq \int_{\Omega} w\left(d d^{c} u\right)^{n} .
$$

Since $\Omega$ is hyperconvex, for any compact $K \subset \Omega$ we can find a function $w \in P S H_{-}(\Omega)$ such that $-1 \leq w$ on $\Omega$ and $w \equiv-1$ on $K$. If we can 
apply the above inequality to $u_{j}=G_{S_{\varepsilon_{j}}}$ and $u=g$, we find the desired inequality.

It remains to see that all those functions belong to Cegrell's class $\mathcal{F}(\Omega)$, as defined in [5, Definition 4.6], or in [6]. Recall that $\mathcal{E}_{0}$ is the class of all bounded functions $v \in P S H(\Omega)$ which tend to 0 at the boundary and such that $\int\left(d d^{c} v\right)^{n}<\infty$. Then $u \in \mathcal{F}(\Omega)$ if and only if there exists a sequence $u_{j}$ decreasing to $u$ with $u_{j} \in \mathcal{E}_{0}$ and $\sup _{j} \int\left(d d^{c} u_{j}\right)^{n}<\infty$.

By Lemma 4.1, for $j$ large enough, $G_{S_{\varepsilon_{j}}} \geq(N+\delta) G_{0}(z)$, and therefore their weak limit $g$ must satisfy the same inequality (almost everywhere). Hence, for any natural number $m, \max (-m, g)$ and $\max \left(-m, G_{S_{\varepsilon_{j}}}\right)$ have bounded Monge Ampère mass, with the same bound, and vanish on the boundary of $\Omega$. Since $g$ (resp. $G_{S_{\varepsilon_{j}}}$ ) is the decreasing limit of $\max (-m, g)\left(\right.$ resp. $\left.\max \left(-m, G_{S_{\varepsilon_{j}}}\right)\right)$, those functions do belong to the class $\mathcal{F}(\Omega)$.

4.2. Inequalities involving limit ideals. Let $\mathcal{I}_{*}:=\liminf _{\varepsilon \rightarrow 0} \mathcal{I}_{\varepsilon}$. We make the additional assumption that $V\left(\mathcal{I}_{*}\right)=\{0\}$. A general lower bound can then be expressed in terms of $\mathcal{I}_{*}$.

Lemma 4.2. For any $\eta>0$ and any neighborhood $\omega$ of 0 , there exists $\varepsilon_{0}>0$ such that for $|\varepsilon|<\varepsilon_{0}$,

$$
G_{\mathcal{I}_{\varepsilon}}(z) \geq\left(e\left(\mathcal{I}_{*}\right)+\eta\right) G_{0}(z), \text { for } z \in \Omega \backslash \omega .
$$

In particular, $\lim \inf _{\varepsilon \rightarrow 0} G_{\mathcal{I}_{\varepsilon}} \geq e\left(\mathcal{I}_{*}\right) G_{0}$.

In order to prove this Lemma, we need to use the notion of integral closure of an ideal.

Definition 4.3. The integral closure $\overline{\mathcal{I}}$ of an ideal $\mathcal{I}$ of a ring $\mathcal{A}$ is the set of all $f \in \mathcal{A}$ such that there exist $m \in \mathbb{N}^{*}$ and $a_{i} \in \mathcal{I}^{m-i}$, $0 \leq i \leq m-1$ such that

$$
f^{m}+\sum_{i=0}^{m-1} a_{i} f^{i}=0 .
$$

We say that an ideal $\mathcal{J} \subset \mathcal{I}$ is a reduction of $\mathcal{I}$ if and only if $\overline{\mathcal{J}}=\overline{\mathcal{I}}$.

It follows from the Briançon-Skoda Theorem [3] that

$$
\overline{\mathcal{I}}=\left\{u \in \mathcal{O}(\Omega):|u| \leq C \max _{i}\left|\psi_{a, i}\right|, \text { near each } a \in \Omega\right\},
$$

see e.g. [11, Corollary 10.5].

Thus $G_{\overline{\mathcal{I}}}^{\Omega}=G_{\mathcal{I}}^{\Omega}$. This provides many examples of distinct ideals with the same Green function, and is at the root of the phenomena of non-convergence. 
Proof of Lemma 4.2.

There exists a reduction $\mathcal{J}$ of $\mathcal{I}_{*}$ generated by exactly $n$ functions, say $f^{1}, \ldots, f^{n} \in \mathcal{O}(\Omega)$ (see e.g. [11, Chapter VIII, Lemma 10.3, p. 394]). Let $f:=\left(f^{1}, \ldots, f^{n}\right)$. Since $G_{\mathcal{J}}=G_{\overline{\mathcal{J}}}=G_{\mathcal{I}_{*}}$, [24, Theorem 2.5] implies $G_{\mathcal{I}_{*}}=\log \|f\|+O(1)$, and the multiplicity of the mapping $f$ at 0 equals $e\left(\mathcal{I}_{*}\right)$.

By definition of $\liminf _{\varepsilon \rightarrow 0} \mathcal{I}_{\varepsilon}$, there are functions $f_{\varepsilon}^{j}$ tending to $f^{j}$ locally uniformly on $\Omega$, for $1 \leq j \leq n$. Then let

$$
\mathcal{J}_{\varepsilon}:=\left\langle f_{\varepsilon}^{1}, \ldots, f_{\varepsilon}^{n}\right\rangle \subset \mathcal{I}_{\varepsilon} .
$$

Let $m_{\varepsilon, k}$ be the multiplicity at the point $a_{\varepsilon, k} \in V\left(\mathcal{J}_{\varepsilon}\right)$ of the mapping $f_{\varepsilon}$. Therefore, using for instance [9, Chap. 2, Theorem 1, p. 60] (statement 8),

$$
\mathcal{J}_{\varepsilon} \supset \cap_{k} \mathfrak{M}_{a_{\varepsilon, k}}^{m_{\varepsilon, k}}=: \mathcal{K}_{\varepsilon}
$$

which implies

$$
G_{\mathcal{I}_{\varepsilon}} \geq G_{\mathcal{J}_{\varepsilon}} \geq G_{\mathcal{K}_{\varepsilon}} \geq \sum_{k} m_{\varepsilon, k} G_{a_{\varepsilon, k}}
$$

By Rouché's theorem (see e.g. [28, §5.2]), when $|\varepsilon|<\varepsilon_{0}, \sum_{k} m_{\varepsilon, k} \leq$ $e\left(\mathcal{I}_{*}\right)$. Since each $G_{a_{\varepsilon, k}} \rightarrow G_{0}$ uniformly on $\Omega \backslash \omega$, we have

$$
G_{a_{\varepsilon, k}} \geq\left(1+\frac{\eta}{e\left(\mathcal{I}_{*}\right)}\right) G_{0}
$$

on $\Omega \backslash \omega$ for $|\varepsilon|<\varepsilon_{0}$ and so,

$$
G_{\mathcal{I}_{\varepsilon}} \geq \sum_{k} m_{\varepsilon, k}\left(1+\frac{\eta}{e\left(\mathcal{I}_{*}\right)}\right) G_{0} \geq\left(e\left(\mathcal{I}_{*}\right)+\eta\right) G_{0} .
$$

\section{Proof of Proposition 1.5.}

As in the proof of Lemma 4.2 above, if $\mathcal{J}$ is a reduction of $\mathcal{I}_{*}$ and $\mathcal{J}=\left\langle f^{1}, \ldots, f^{n}\right\rangle$,

$$
G_{\mathcal{I}_{*}}=\log \|f\|+O(1) .
$$

Given some $\Omega^{\prime}$ relatively compact in $\Omega$, we can assume $\sup _{\Omega^{\prime}}\|f\|<1$. Then for $|\varepsilon|<\varepsilon_{0}, \sup _{\Omega^{\prime}}\left\|f_{\varepsilon}\right\|<1$, where $f_{\varepsilon}=\left(f_{\varepsilon}^{1}, \ldots, f_{\varepsilon}^{n}\right)$, again as above. Thus for $z \in \Omega^{\prime}$,

$$
\log \left\|f_{\varepsilon}(z)\right\| \leq G_{\mathcal{I}_{\varepsilon}}^{\Omega^{\prime}}(z) .
$$

By Lemma 4.2, for all $z \in \partial \Omega^{\prime}$, for $|\varepsilon|<\varepsilon_{0}$,

$$
G_{\mathcal{I}_{\varepsilon}}(z) \geq\left(e\left(\mathcal{I}_{*}\right)+1\right) \inf _{\Omega \backslash \Omega^{\prime}} G_{0}(z)=: A .
$$


Since $G_{\mathcal{I}_{\varepsilon}}-A$ is maximal on $\Omega \backslash\{0\}$, nonnegative on $\partial \Omega^{\prime}$ and $G_{\mathcal{I}_{\varepsilon}}-A=$ $G_{\mathcal{I}_{\varepsilon}}^{\Omega^{\prime}}+O(1)$ near 0 , we have

$$
G_{\mathcal{I}_{\varepsilon}}-A \geq G_{\mathcal{I}_{\varepsilon}}^{\Omega^{\prime}}
$$

on $\Omega^{\prime}$, so (4.2) gives, for $z \in \Omega^{\prime}$,

$$
\log \left\|f_{\varepsilon}(z)\right\|+A \leq G_{\mathcal{I}_{\varepsilon}}(z) .
$$

Since $f_{\varepsilon}$ converges to $f$ uniformly on $\Omega^{\prime}, \log \|f(z)\|+A \leq \liminf _{\varepsilon \rightarrow 0} G_{\mathcal{I}_{\varepsilon}}(z)$ on $\Omega^{\prime}$, so (4.1) implies $G_{\mathcal{I}_{*}} \leq \liminf _{\varepsilon \rightarrow 0} G_{\mathcal{I}_{\varepsilon}}(z)+O(1)$.

Remark. If $G_{\mathcal{I}_{\varepsilon}}$ converges uniformly to $g$ on compact subsets of $\Omega \backslash\{0\}$, then Proposition 1.5 implies that $G_{\mathcal{I}_{*}} \leq g$.

4.3. Uniform convergence. We start with a sufficient condition for uniform convergence that will be useful in particular in Section 6. We need a bit of shorthand from [7].

Definition 4.4. Let $u_{1}, u_{2} \in P S H(\Omega)$, such that $u_{1}^{-1}\{-\infty\}=u_{2}^{-1}\{-\infty\}=$ $\{0\}$. We say that $u_{1}$ and $u_{2}$ are equivalent near 0 , and we write $u_{1} \sim_{0} u_{2}$, if and only if there exists a neighborhood $U$ of 0 such that $u_{1}-\left.u_{2}\right|_{U} \in L^{\infty}(U)$.

This implies that $\left(d d^{c} u_{1}\right)^{n}(\{0\})=\left(d d^{c} u_{2}\right)^{n}(\{0\})$.

Lemma 4.5. Suppose that there exists a function $G$ from $\Omega$ to $[-\infty, 0]$ and a constant $C>0$ such that for any $\delta \in\left(0, \delta_{0}\right]$, there exists $\varepsilon(\delta)>0$ such that for any $\varepsilon$ with $|\varepsilon|<\varepsilon(\delta)$, for any $z$ such that $\|z\|=\delta$,

$$
\left|G_{S_{\varepsilon}}(z)-G(z)\right| \leq C \text {. }
$$

Then $\lim _{\varepsilon \rightarrow 0} G_{S_{\varepsilon}}(z)=g(z)$, uniformly on compacta of $\Omega \backslash\{0\}$, and clearly $g \sim_{0} G$.

Proof. It is enough to see that for any $\delta_{0}>0$, for any $\eta>0$, there exists $\varepsilon_{0}>0$ such that for any $\varepsilon_{1}, \varepsilon_{2}$ with $\left|\varepsilon_{1}\right|,\left|\varepsilon_{2}\right|<\varepsilon_{0}$, for any $z \in$ $\Omega \backslash B\left(0, \delta_{0}\right)$,

$$
(1+\eta) G_{S_{\varepsilon_{1}}}(z) \leq G_{S_{\varepsilon_{2}}}(z) \leq(1-\eta) G_{S_{\varepsilon_{1}}}(z) .
$$

By the hypothesis, and by Lemma 4.1, $G(z) \leq \frac{1}{2} G_{0}(z)+C$. Therefore there exists $\delta_{1} \in\left(0, \delta_{0}\right)$ such that for $\|z\|=\delta<\delta_{1}$,

$$
(1+\eta) G(z) \leq G(z)-C<G(z)+C \leq(1-\eta) G(z) .
$$

Using the hypothesis once again, there exists $\varepsilon(\delta)>0$ such that for any $\varepsilon$ with $|\varepsilon|<\varepsilon(\delta)$, for any $z$ such that $\|z\|=\delta$, (4.3) holds. Both functions $G_{S_{\varepsilon_{1}}}$ and $G_{S_{\varepsilon_{2}}}$ are maximal plurisubharmonic, and equal to 0 on $\partial \Omega$, so those inequalities extend to $\Omega \backslash B(0, \delta) \supset \Omega \backslash B\left(0, \delta_{0}\right)$. 
Proof of Propositions 1.2 and 1.6.

Denote $\mathcal{I}^{*}:=\lim \sup _{\varepsilon \rightarrow 0} \mathcal{I}_{\varepsilon}$. Let $h \in \mathcal{I}^{*}, \sup _{\Omega^{\prime}}|h|<1$; let $\left(h_{\varepsilon_{j}}\right)_{j}$ be a sequence of holomorphic functions converging uniformly to $h$ such that $h_{\varepsilon_{j}} \in \mathcal{I}_{\varepsilon_{j}}$. Then $\log \left|h_{\varepsilon_{j}}\right| \leq G_{\mathcal{I}_{\varepsilon_{j}}}^{\Omega^{\prime}}$ on $\Omega^{\prime}$ and so, as in the proof of Proposition 1.5,

$$
\log \left|h_{\varepsilon_{j}}\right| \leq G_{\mathcal{I}_{\varepsilon_{j}}}^{\Omega}+A .
$$

Therefore $\log |h| \leq g+A$ and thus, applying this to any generator of $\mathcal{I}^{*}, G_{\mathcal{I}^{*}} \leq g$.

Proposition 1.2 then follows as a special case.

Proof of Corollary 1.3. Let $g:=\lim _{\varepsilon \rightarrow 0} G_{\mathcal{I}_{\varepsilon}}, \mathcal{I}:=\lim _{\varepsilon \rightarrow 0} \mathcal{I}_{\varepsilon}$. Since we have uniform convergence on any compact subset of $\Omega \backslash \omega$, it is easy to show that $g$ also is maximal plurisubharmonic on any such compactum, and therefore on the whole of $\Omega \backslash\{0\}$. So $\left(d d^{c} g\right)^{n}=\mu \delta_{0}$, and by Proposition 1.13 ,

$\mu=\left(d d^{c} g\right)^{n}(\Omega) \leq \liminf _{\varepsilon \rightarrow 0}\left(d d^{c} G_{\mathcal{I}_{\varepsilon}}\right)^{n}(\Omega)=N=\ell(\mathcal{I}) \leq e(\mathcal{I})=\left(d d^{c} G_{\mathcal{I}}\right)^{n}(\Omega)$,

which implies the claimed inequality between the Monge-Ampère measures.

\section{Convergence And Non-Convergence}

\subsection{Proof of Theorem 1.8.}

Since the family $\left(\left\|\Psi_{\varepsilon}\right\|\right)_{\varepsilon}$ is bounded on $\bar{\Omega}$, there is a constant $C$ such that $\log \left\|\Psi_{\varepsilon}\right\|-C \in P S H_{-}(\Omega)$, and it admits logarithmic singularities at the points $a_{j}^{\varepsilon}$, thus $\log \left\|\Psi_{\varepsilon}\right\|-C \leq G_{\varepsilon}$.

Since $\Psi_{\varepsilon}$ has $n$ components, it is well-known that $\log \left\|\Psi_{\varepsilon}\right\|$ is a maximal plurisubharmonic function on $\Omega \backslash S_{\varepsilon}$ (by composition with the holomorphic map, it is enough to check it for $u(z):=\log \|z\|^{2}$, an elementary computation). If $a \in \Psi_{0}^{-1}\{0\}$, then for any small enough $\delta>0,0<\min _{\partial B(a, \delta)}\left\|\Psi_{0}\right\|$, so if $\Psi_{\varepsilon}^{-1}\{0\} \cap B(a, \delta)$ was empty, uniform convergence and maximality of $\log \left\|\Psi_{\varepsilon}\right\|$ would imply that

$$
\min _{\bar{B}(a, \delta)} \log \left\|\Psi_{\varepsilon}\right\|>-1+\log \left(\min _{\partial B(a, \delta)}\left\|\Psi_{0}\right\|\right)>-\infty
$$

for $|\varepsilon|$ small enough, which contradicts $\lim \left\|\Psi_{\varepsilon}(a)\right\|=0$.

Since all the points of $\Psi_{\varepsilon}^{-1}\{0\}$ tend to 0 , we have $\Psi_{0}^{-1}\{0\}=\{0\}$, and for any compact $K \subset \bar{\Omega} \backslash\{0\}$, any $\varepsilon$ close enough to $0, \min _{K}\left\|\Psi_{\varepsilon}\right\| \geq$ $c_{K}>0$, thus $G_{\varepsilon} \leq \log \left\|\Psi_{\varepsilon}\right\|-\log c_{K}$. Since $G_{S}^{\Omega}(z)=-\infty$ iff $z \in S$, and $G_{S}(z) \leq \log \|z-s\|+O(1)$ in a neighborhood of each $s \in S$ [10], [18], using [24, Lemma 4.1], we see that

$$
G_{\varepsilon} \leq \log \left\|\Psi_{\varepsilon}\right\|+O(1),
$$


where the $O(1)$ is independent of $\varepsilon$.

On the other hand, by the very definition of $\mathcal{I}_{0}$ and of its Green function, $G_{\mathcal{I}_{0}} \geq \log \left\|\Psi_{0}\right\|+O(1)$. By [24, Theorem 2.5], $G_{\mathcal{I}_{0}} \leq \log \left\|\Psi_{0}\right\|+$ $O(1)$ in a neighborhood of 0 , and again [24, Lemma 4.1] shows that

$$
G_{\mathcal{I}_{0}} \leq \log \left\|\Psi_{0}\right\|+O(1) \text { on } \Omega .
$$

The hypothesis of uniform convergence of the $\Psi_{\varepsilon}^{j}$ shows that we can apply Lemma 4.5 with $G=\log \left\|\Psi_{0}\right\|$, therefore $\lim _{\varepsilon \rightarrow 0} G_{\varepsilon}=g=$ $\log \left\|\Psi_{0}\right\|+O(1)$, with uniform convergence on compacta of $\bar{\Omega} \backslash\{0\}$. Furthermore, $\left.g\right|_{\partial \Omega}=0$, and the uniform convergence implies that $g$ is maximal plurisubharmonic on $\Omega \backslash\{0\}$, so we can apply [24, Lemma 4.1] in both directions to conclude that $g=G_{\mathcal{I}_{0}}$, which proves the second statement in Theorem 1.8 .

Now we prove the statement about ideals. For any $f \in \mathcal{I}_{0}$,

$$
f=\sum_{i=1}^{n} h_{i} \Psi_{0}^{i}=\lim _{\varepsilon \rightarrow 0} \sum_{i=1}^{n} h_{i} \Psi_{\varepsilon}^{i},
$$

with uniform convergence on compacta, so that $\mathcal{I}_{0} \subset \liminf \inf _{\varepsilon \rightarrow 0} \mathcal{I}_{\varepsilon}$.

To prove the reverse inclusion, we need to use the characterization of an ideal $\mathcal{I}_{\Psi}:=\left\langle\Psi^{1}, \ldots, \Psi^{n}\right\rangle$ by multidimensional residues. For simplicity, we assume that $\Psi^{-1}(0) \cap \Omega=\{0\}$, so that a holomorphic function belongs to $\mathcal{I}_{\Psi}$ if and only if its germ at 0 is in the ideal of germs with the same generators, $\mathcal{I}_{\Psi, 0}$ (recall that $\Omega$ is contractible and bounded). We take the next definition from [28, §5.1, p. 14].

Definition 5.1. Let $\Psi$ be a holomorphic mapping $\bar{\omega} \longrightarrow \mathbb{C}^{n}$ where $\omega$ is a bounded neighborhood of 0 in $\mathbb{C}^{n}$, with $\Psi^{-1}(0) \cap \bar{\omega}=\{0\}$. We choose a real $n$-dimensional chain

$$
\Gamma=\Gamma^{\delta}(\Psi):=\left\{z \in \omega:\left|\Psi^{j}\right|=\delta_{j}, 1 \leq j \leq n\right\},
$$

where $\delta_{j}>0$ are small enough so that $\Gamma^{\delta}(\Psi)$ be relatively compact in $\omega$, with its orientation determined by the condition $d\left(\arg \Psi_{1}\right) \wedge \cdots \wedge$ $d\left(\arg \Psi_{n}\right) \geq 0$ (by Sard's lemma, we can choose values of $\delta$ such that $\Gamma$ is smooth). Let $h$ be holomorphic on $\omega$. The local residue of $h$ at the point 0 is

$$
r e s_{0, \Psi}(h):=\frac{1}{(2 \pi i)^{n}} \int_{\Gamma} \frac{h d z_{1} \wedge \cdots \wedge d z_{n}}{\Psi^{1} \cdots \Psi^{n}} .
$$

This residue is well-defined in the sense that it does not depend on the choice of a particular $\Gamma$ [28, p. 15]. In fact it can be computed by integration on $\partial \omega$. We omit the formula, but a consequence is the "continuity principle" [28, §5.4, Proposition, p. 20]. We apply it to 
the situation of Theorem 1.8. By the hypothesis on $\Psi_{\varepsilon}$, for $|\varepsilon| \leq \varepsilon_{0}$, $S_{\varepsilon}=\Psi_{\varepsilon}^{-1}(0) \subset \omega$ and is made up of isolated zeroes.

Proposition 5.2. Let $h$ be holomorphic in a neighborhood of $\bar{\omega}$, then

$$
\lim _{\varepsilon \rightarrow 0} \sum_{p \in S_{\varepsilon}} r e s_{p, \Psi_{\varepsilon}} h=r e s_{0, \Psi_{0}} h .
$$

The characterization of the ideal $\mathcal{I}_{\Psi}$ follows immediately from the Local Duality Theorem [28, §5.6, p. 23].

Theorem 5.3. A germ $h$ of holomorphic function belongs to the ideal $\mathcal{I}_{\Psi, 0}$ of germs of functions at 0 generated by the components of $\Psi$ if and only if for any holomorphic germ $g$ at 0 , res $s_{0, \Psi}(h g)=0$.

We return to the proof. Suppose that $f \in \mathcal{O}(\Omega)$ and there exists sequences $\left(f_{j}\right) \subset \mathcal{O}(\Omega)$ and $\varepsilon_{j} \rightarrow 0$ such that $f_{j} \in \mathcal{I}_{\varepsilon_{j}}, f_{j} \rightarrow f$ as $j \rightarrow \infty$, uniformly on compacta of $\Omega$. Take any holomorphic germ $g$ at 0 , and $\omega$ a neighborhood of 0 small enough so that $g$ is holomorphic in a neighborhood of $\bar{\omega}$ and we can apply Proposition 5.2. Then for any $p \in S_{\varepsilon_{j}}, \operatorname{res}_{p, \Psi_{\varepsilon_{j}}} f_{j} g=0$ by the "only if" part of Theorem 5.3, therefore $\operatorname{res}_{p, \Psi_{0}} f g=\lim _{j \rightarrow \infty} 0$ by Proposition 5.2 , and the "if" part of Theorem 5.3 shows that $f \in \mathcal{I}_{0}$.

\subsection{Proof of Theorem 1.11.}

In the "only if" direction, we prove a slightly stronger statement than in the Theorem: we only assume that $g=\lim _{\varepsilon \rightarrow 0} G_{\mathcal{I}_{\varepsilon}}$ in $L_{l o c}^{1}$, and we don't assume that $\left(\mathcal{I}_{\varepsilon}\right)_{\varepsilon}$ converges. Write $\mathcal{I}=\liminf _{\varepsilon \rightarrow 0} \mathcal{I}_{\varepsilon}$. Then, by Lemma $2.2, N \leq \ell(\mathcal{I})$. On the other hand, by Proposition 1.13, $\left(d d^{c} g\right)^{n}(\Omega) \leq \liminf _{\varepsilon \rightarrow 0}\left(d d^{c} G_{\varepsilon}\right)^{n}(\Omega)=N$.

On the other hand, the Hilbert-Samuel multiplicity of an ideal, which is invariant under integral closure [11, Theorem 10.4 (b)], like its Green function, is indeed related to it.

Proposition 5.4. If $V(\mathcal{I})=\{a\},\left(d d^{c} G_{\mathcal{I}}\right)^{n}=e(\mathcal{I}) \delta_{a}$.

A proof can be found for instance in [12, Lemma 2.1, p. 4].

Now suppose that $\ell(\mathcal{I})<e(\mathcal{I})$. Then

$$
\left(d d^{c} g\right)^{n}(\Omega) \leq N \leq \ell(\mathcal{I})<e(\mathcal{I})=\left(d d^{c} G_{\mathcal{I}}\right)^{n}(\Omega),
$$

thus $g \neq G_{\mathcal{I}}$.

Note that the same proof can be done when $\varepsilon$ tends to 0 along a subset. We omit the details.

Conversely, let $\psi_{1}, \ldots, \psi_{n}$ be generators of $\mathcal{I}$; we may assume them to be defined on a neighborhood of $\bar{\Omega}$. Since $\mathcal{I}_{\varepsilon} \rightarrow \mathcal{I}$, for any $\varepsilon \in$ $\left(0, \varepsilon_{0}\right)$ there exist functions $\psi_{k, \varepsilon} \in \mathcal{I}_{\varepsilon}$ converging to $\psi_{k}$ uniformly on 
compacts of $\Omega, 1 \leq k \leq n$. Then by Rouché's theorem (see e.g. [28, §5.2, Proposition 3, p. 16]), each mapping $\Psi_{\varepsilon}=\left(\psi_{1, \varepsilon}, \ldots, \psi_{n, \varepsilon}\right)$ has isolated zeros, say at points $a_{j}(\varepsilon), 1 \leq j \leq N(\varepsilon)$, and their total number, counted with the corresponding multiplicities $m_{j}(\varepsilon)$, equals the multiplicity of the mapping $\Psi=\left(\psi_{1}, \ldots, \psi_{n}\right)$ at 0 . The latter equals $N$ because having $n$ generators is equivalent to the condition $e(\mathcal{I})=\ell(\mathcal{I})$, and $\ell(\mathcal{I})=\lim \ell\left(\mathcal{I}_{\varepsilon}\right)=N$ by Lemmas 2.1, 2.2.

By construction, $\mathcal{I}_{\Psi_{\varepsilon}} \subseteq \mathcal{I}_{\varepsilon}$, and

$$
\ell\left(\mathcal{I}_{\Psi_{\varepsilon}}\right) \leq \sum_{j} \ell\left(\left(\mathcal{I}_{\Psi_{\varepsilon}}\right)_{a_{j}(\varepsilon)}\right) \leq N=\ell\left(\mathcal{I}_{\varepsilon}\right)
$$

so both ideals coincide. Therefore $G_{\varepsilon}=G_{\mathcal{I}_{\varepsilon}}=G_{\mathcal{I}_{\Psi_{\varepsilon}}}$ and the family $\mathcal{I}_{\Psi_{\varepsilon}}$ satisfies the Uniform Complete Intersection condition, so an application of Theorem 1.8 completes the proof.

\section{EXAMPLE : TWO AND THREE POINTS SYSTEMS}

6.1. The case of two points. We begin by sketching what happens in the case where $S_{\varepsilon}=\left\{p_{1}(\varepsilon), p_{2}(\varepsilon)\right\}$ (two distinct points), with $\lim _{\varepsilon \rightarrow 0} p_{1}(\varepsilon)=\lim _{\varepsilon \rightarrow 0} p_{2}(\varepsilon)=0 \in \Omega$. By compactness, there is a subsequence $\varepsilon_{j}$ such that $\left[p_{1}\left(\varepsilon_{j}\right)-p_{2}\left(\varepsilon_{j}\right)\right] \rightarrow \nu \in \mathbb{P}^{n-1} \mathbb{C}$, where $[z]$ denotes the class of $z$ in $\mathbb{P}^{n-1} \mathbb{C}$, for $z \in \mathbb{C}^{n} \backslash\{0\}$. From now on we assume that $\varepsilon \in\left\{\varepsilon_{j}\right\}$ and drop the subscript. By applying translations tending to 0 , we may assume that $p_{1}(\varepsilon)=0$.

Pick orthonormal bases $\mathcal{B}:=\left(v_{1}, \ldots, v_{n}\right)$ such that $\left[v_{1}\right]=\nu$, and $\mathcal{B}^{\varepsilon}:=\left(v_{1}^{\varepsilon}, \ldots, v_{n}^{\varepsilon}\right)$ tending to $\left(v_{1}, \ldots, v_{n}\right)$ such that $\left[p_{2}(\varepsilon)\right]=\left[v_{1}^{\varepsilon}\right]$, so that $p_{2}(\varepsilon)=\rho_{\varepsilon} v_{1}^{\varepsilon}, \rho_{\varepsilon} \rightarrow 0$. Let $\left(\xi_{1}, \ldots, \xi_{n}\right)$, resp. $\left(\xi_{1}^{\varepsilon}, \ldots, \xi_{n}^{\varepsilon}\right)$ stand for the coordinates in $\mathcal{B}$, resp. $\mathcal{B}^{\varepsilon}$. Finally, for $R>0, D_{R}:=\{z$ : $\left.\max \left|\xi_{j}\right|<R\right\}, D_{R}^{\varepsilon}:=\left\{z: \max \left|\xi_{j}^{\varepsilon}\right|<R\right\}$.

Lemma 6.1. [15] If $S_{i} \subset \Omega_{i} \subset \mathbb{C}^{n_{i}}, i=1,2$, then

$$
G_{S_{1} \times S_{2}}^{\Omega_{1} \times \Omega_{2}}\left(z_{1}, z_{2}\right)=\max \left(G_{S_{1}}^{\Omega_{1}}\left(z_{1}\right), G_{S_{2}}^{\Omega_{2}}\left(z_{2}\right)\right) .
$$

Thus

$$
G_{S_{\varepsilon}}^{D_{R}^{\varepsilon}}(z)=\max \left(\log \left|\frac{\xi_{1}^{\varepsilon}\left(\rho_{\varepsilon}-\xi_{1}^{\varepsilon}\right)}{R^{2}-\xi_{1}^{\varepsilon} \bar{\rho}_{\varepsilon}}\right|, \log \left|\frac{\xi_{j}^{\varepsilon}}{R}\right|, 2 \leq j \leq n\right) .
$$

There are $0<R_{1}<R_{2}$ such that for $|\varepsilon|$ small enough, $D_{R_{1}}^{\varepsilon} \subset \Omega \subset D_{R_{2}}^{\varepsilon}$, so

$$
G_{S_{\varepsilon}}^{D_{R_{1}}^{\varepsilon}}(z) \geq G_{S_{\varepsilon}}^{\Omega}(z) \geq G_{S_{\varepsilon}}^{D_{R_{2}}^{\varepsilon}}(z)
$$

The above inequality and an elementary computation show that, setting $g(z):=\max \left(\log \left|\xi_{1}\right|^{2}, \log \left|\xi_{j}\right|, 2 \leq j \leq n\right)$, there are constants 
$C_{1}, C_{2} \in \mathbb{R}$ such that if $z \in \Omega \backslash B\left(0, r_{0}\right)$ and $|\varepsilon|<\varepsilon_{0}\left(r_{0}\right)$,

$$
g(z)+C_{1} \leq G_{S_{\varepsilon}}^{\Omega}(z) \leq g(z)+C_{2} .
$$

By Lemma 4.5, $G_{S_{\varepsilon}}^{\Omega}$ converges locally uniformly on $\Omega \backslash\{0\}$ to $G=$ $g+O(1)$. This means in particular that $G\left(\zeta v_{1}\right)=2 \log |\zeta|+O(1)$, while for any vector $v$ not colinear to $v_{1}, G(\zeta v)=\log |\zeta|+O(1)$. As a consequence, if there are two subsequences $\left\{\varepsilon_{j}\right\},\left\{\tilde{\varepsilon}_{j}\right\}$ such that $v_{1} \neq \tilde{v}_{1}$ (with the obvious notation), then $G_{S_{\varepsilon}}^{\Omega}$ doesn't admit any limit, while if $\lim _{\varepsilon \rightarrow 0}\left[p_{2}(\varepsilon)\right]$ exists (i.e. any convergent subsequence converges to the same limit), then $\lim _{\varepsilon \rightarrow 0} G_{S_{\varepsilon}}^{\Omega}=G=g+O(1)$, with local uniform convergence. This necessary and sufficient condition for convergence can be expressed by saying that $p_{2}^{\varepsilon}$ converges in the blow-up at 0 of $\mathbb{C}^{n}$. It is the same as for convergence of two-point Lempert functions [27, Theorem 3.3].

6.2. When all directions coincide. Proof of Theorem 1.12, part (2). Consider the following family of examples, where $\alpha \in \mathbb{C}$ :

$$
S_{\varepsilon}:=\left\{(0,0) ;\left(\varepsilon, \alpha \varepsilon^{2}\right) ;\left(-\varepsilon, \alpha \varepsilon^{2}\right)\right\} .
$$

The ideal of functions vanishing on $S_{\varepsilon}$ is clearly generated by $\left\{z_{1}\left(z_{1}^{2}-\right.\right.$ $\left.\left.\varepsilon^{2}\right), z_{2}-\alpha z_{1}^{2}\right\}$, so its limiting ideal is $<z_{1}^{3}, z_{2}-\alpha z_{1}^{2}>$ which depends on $\alpha$. This is a case where the Uniform Complete Intersection condition holds, and the limiting Green functions will be equivalent to $\max \left(3 \log \left|z_{1}\right|, \log \left|z_{2}-\alpha z_{1}^{2}\right|\right)$, so they are distinct for distinct values of $\alpha$.

6.3. Three points, two directions : limit ideal. To prove the first part of Theorem 1.12, we first deal with the statement about convergence of ideals.

Consider a function holomorphic in a neighborhood of the origin in $\mathbb{C}^{n}$ vanishing in two points $a, b$, close enough to 0 . Renormalizing if needed, we assume that $f$ is holomorphic on the ball $B(a, 1)$. Let $d:=\|a-b\|, v:=\frac{1}{d}(b-a), f_{a, b}(\zeta):=f(a+\zeta v) \in \mathcal{O}(\overline{\mathbb{D}})$. Then

$$
\int_{\partial \mathbb{D}} \frac{f_{a, b}(\zeta)}{\zeta(\zeta-d)} d \zeta=0
$$

so if we have a sequence $f_{\varepsilon}$ vanishing on $a_{\varepsilon}, b_{\varepsilon} \rightarrow 0$, and converging uniformly on compacta to $f$, and if $v_{\varepsilon} \rightarrow v$, then $f(0,0)=0$ and

$$
\int_{\partial \mathbb{D}} \frac{f(\zeta v)}{\zeta^{2}} d \zeta=0
$$

thus $\partial_{v} f(0,0)=0$. This is another special case of the first statement of Theorem [1.8. In case (i) of Theorem 1.12, we may assume 
$i=3, j=2$ and perform this reasoning with $\left(a_{\varepsilon}, b_{\varepsilon}\right)=\left(a_{1}^{\varepsilon}, a_{2}^{\varepsilon}\right)$ and $\left(a_{\varepsilon}, b_{\varepsilon}\right)=\left(a_{1}^{\varepsilon}, a_{3}^{\varepsilon}\right)$; the relevant vectors converge to two independent directions, $v_{3}$ and $v_{2}$ respectively, we are in dimension 2 , therefore all first partial derivatives of $f$ must vanish at the origin. This means that $\lim \sup _{\varepsilon \rightarrow 0} \mathcal{I}_{\varepsilon} \subset \mathfrak{M}_{0}^{2}$.

To get the converse inclusion, it will be enough to approximate the generators of $\mathfrak{M}_{0}^{2}$. Since this ideal (as well as the notion of convergence) is invariant under invertible linear maps, we may reduce ourselves to the case where $v_{3}=[1: 0]$ and $v_{2}=[0: 1]$. Then $a_{1}^{\varepsilon}=\left(x_{1}(\varepsilon), x_{2}(\varepsilon)\right)$, where $x_{j}(\varepsilon)=o(1), j=1,2$; and $a_{2}^{\varepsilon}-a_{1}^{\varepsilon}=\left(\rho_{2}(\varepsilon), \delta_{2}(\varepsilon)\right), a_{3}^{\varepsilon}-a_{1}^{\varepsilon}=$ $\left(\delta_{3}(\varepsilon), \rho_{3}(\varepsilon)\right)$, where $\delta_{j}(\varepsilon)=o\left(\rho_{j}(\varepsilon)\right), j=2,3$. Then

$$
\begin{gathered}
z_{1}^{2}=\lim _{\varepsilon \rightarrow 0}\left(z_{1}-x_{1}(\varepsilon)-\rho_{2}(\varepsilon)\right)\left(z_{1}-x_{1}(\varepsilon)-\frac{\delta_{3}(\varepsilon)}{\rho_{3}(\varepsilon)}\left(z_{2}-x_{2}(\varepsilon)\right)\right) \in \liminf _{\varepsilon \rightarrow 0} \mathcal{I}_{\varepsilon}, \\
z_{1} z_{2}=\lim _{\varepsilon \rightarrow 0}\left(z_{1}-x_{1}(\varepsilon)-\frac{\delta_{3}(\varepsilon)}{\rho_{3}(\varepsilon)}\left(z_{2}-x_{2}(\varepsilon)\right)\right)\left(z_{2}-x_{2}(\varepsilon)-\frac{\delta_{2}(\varepsilon)}{\rho_{2}(\varepsilon)}\left(z_{1}-x_{1}(\varepsilon)\right)\right) \\
\in \liminf _{\varepsilon \rightarrow 0} \mathcal{I}_{\varepsilon},
\end{gathered}
$$

and a similar computation takes care of $z_{2}^{2}$.

6.4. First reduction : changing the axes. The rest of this section is devoted to the more intricate proof of the convergence of Green functions, which will be carried out by reduction to two model cases, given in the proposition below. We use the notation from Definition 4.4 .

\subsubsection{Model Cases.}

Proposition 6.2. With the hypotheses and notations of Theorem 1.12, suppose furthermore that $\Omega=\mathbb{D}^{2}$ and that there are functions $\delta_{i}(\varepsilon)$ with $\delta_{i}(\varepsilon) / \varepsilon \rightarrow 0,1 \leq i \leq 4$ and $\omega(\varepsilon) \rightarrow 0$ such that

$$
a_{1}^{\varepsilon}=(0,0), \quad a_{2}^{\varepsilon}=\left(\varepsilon, \delta_{1}(\varepsilon)\right)
$$

and either

(1) $a_{3}^{\varepsilon}=\left(\delta_{2}(\varepsilon), \varepsilon+\delta_{3}(\varepsilon)\right)$

(2) or $a_{3}^{\varepsilon}=\left(\delta_{4}(\varepsilon) \omega(\varepsilon), \delta_{4}(\varepsilon)\right)$,

then there exists plurisubharmonic functions $g_{1}, g_{2}$ such that in case (i), $i=1,2, \lim _{\varepsilon \rightarrow 0} G_{\varepsilon}=g_{i}$, uniformly on compacta of $\mathbb{D}^{2} \backslash\{0\}$, and $g_{1} \sim_{0} H, g_{2} \sim_{0} F$, where $H$ and $F$ are described below. Consequently, $\left(d d^{c} g_{i}\right)^{2}=3 \delta_{0}, i=1,2$. 
We now construct the auxiliary functions $H(z)$ and $F(z)$. The following gives a partition of the extended complex plane :

$$
\mathcal{D}_{3}:=\bar{D}\left(-1, \frac{1}{2}\right), \quad \mathcal{D}_{1}:=\overline{\mathbb{D}} \backslash \mathcal{D}_{3}, \quad \mathcal{D}_{2}:=\mathbb{C} \cup\{\infty\} \backslash\left(\mathcal{D}_{1} \cup \mathcal{D}_{3}\right) .
$$

We now define a partition of $\mathbb{D}^{2} \backslash\{0\}$.

$$
\begin{gathered}
D_{0}:=\left\{z \in \mathbb{D}^{2} \backslash\{0\}:\left|z_{2}\right| \leq\left|z_{1}\right|^{2} \text {, or }\left|z_{1}\right| \leq\left|z_{2}\right|^{2} \text {, or }\left|z_{1}+z_{2}\right| \leq\left|z_{1}\right|^{2}\right\} \\
D_{j}:=\left\{z \in \mathbb{D}^{2} \backslash\left(D_{0} \cup\{0\}\right): \frac{z_{2}}{z_{1}} \in \mathcal{D}_{j}\right\}, \quad j=1,2,3 .
\end{gathered}
$$

Here is the piecewise definition of our auxiliary function.

$$
H(z):= \begin{cases}\max \left(2 \log \left|z_{1}\right|, 2 \log \left|z_{2}\right|\right) & \text { if } z \in D_{0}, \\ \log \left|z_{1}\right|+\frac{1}{2} \log \left|z_{2}\right| & \text { if } z \in D_{1}, \\ \frac{1}{2} \log \left|z_{1}\right|+\log \left|z_{2}\right| & \text { if } z \in D_{2}, \\ \log \left|z_{1}\right|+\frac{1}{2} \log \left|z_{1}+z_{2}\right| & \text { if } z \in D_{3} .\end{cases}
$$

The definition of the auxiliary function $F$ requires another partition of the bidisk.

$D_{0}^{\prime}:=\left\{z \in \mathbb{D}^{2} \backslash\{0\}:\left|z_{2}\right| \leq\left|z_{1}\right|^{3 / 2}\right.$ or $\left.\left|z_{1}\right| \leq\left|z_{2}\right|^{2}\right\}, \quad D_{1}^{\prime}:=\mathbb{D}^{2} \backslash\left(D_{0}^{\prime} \cup\{0\}\right)$.

$$
F(z):= \begin{cases}\max \left(2 \log \left|z_{1}\right|, 2 \log \left|z_{2}\right|\right) & \text { if } z \in D_{0}^{\prime} \\ \frac{1}{2} \log \left|z_{1}\right|+\log \left|z_{2}\right| & \text { if } z \in D_{1}^{\prime} .\end{cases}
$$

6.4.2. Changing the coordinates. To reduce ourselves to the cases occurring in Proposition 6.2, we will need to make linear changes of coordinates.

Definition 6.3. We say that a function $G: \Omega \rightarrow \mathbb{R}$ is admissible if and only if

$$
\forall R>1, \exists C(R) \text { such that } \forall z \in \Omega \cap \frac{1}{R} \Omega,|G(z)-G(R z)| \leq C(R) .
$$

In particular, $G \sim_{0} G^{\prime}$ for any dilation $G^{\prime}$ of $G$. When $G$ is unbounded, this forces it to have logarithmic growth. We will see that this onerous looking hypothesis is actually satisfied in the examples we are interested in.

Lemma 6.4. Let $\Omega$ be a bounded hyperconvex domain, $0 \in \Omega$. Suppose that $\left(S_{\varepsilon}\right) \subset \Omega$ is such that for any $R>0, \lim _{\varepsilon \rightarrow 0} G_{R S_{\varepsilon}}(z)=g_{R}(z)$, uniformly on compacta of $\Omega \backslash\{0\}$, and that there exists a function $G$ on $\Omega$ such that for any $R>0, g_{R} \sim_{0} G$, and $G$ is admissible. Let $\Phi$ be 
an invertible linear map of $\mathbb{C}^{n}$, and $\Omega_{1}$ a domain satisfying the same hypotheses as $\Omega$.

Then $\lim _{\varepsilon \rightarrow 0} G_{\Phi^{-1}\left(S_{\varepsilon}\right)}^{\Omega_{1}}(z)=\tilde{g}(z)$, uniformly on compacta of $\Omega_{1} \backslash\{0\}$, with $\tilde{g}(z) \sim_{0} g \circ \Phi$.

Proof. Let $0<R_{1}<R_{2}$ be such that $R_{1} \Omega \subset \Phi\left(\Omega_{1}\right) \subset R_{2} \Omega$. Since $G_{\Phi^{-1}\left(S_{\varepsilon}\right)}^{\Omega_{1}}(z)=G_{S_{\varepsilon}}^{\Phi\left(\Omega_{1}\right)}(\Phi(z))$, we have

$G_{R_{2}^{-1} S_{\varepsilon}}^{\Omega}\left(\frac{1}{R_{2}} \Phi(z)\right)=G_{S_{\varepsilon}}^{R_{2} \Omega}(\Phi(z)) \leq G_{\Phi^{-1}\left(S_{\varepsilon}\right)}^{\Omega_{1}}(z) \leq G_{S_{\varepsilon}}^{R_{1} \Omega}(\Phi(z))=G_{R_{1}^{-1} S_{\varepsilon}}^{\Omega}\left(\frac{1}{R_{1}} \Phi(z)\right)$.

Let $\delta>0$. On $\Omega \backslash B(0, \delta)$, for $\varepsilon$ small enough,

$$
G_{\Phi^{-1}\left(S_{\varepsilon}\right)}^{\Omega_{1}}(z) \geq g_{R_{2}^{-1}}\left(\frac{1}{R_{2}} \Phi(z)\right)-1 \geq G\left(\frac{1}{R_{2}} \Phi(z)\right)-C_{1} \geq G(\Phi(z))-C_{2},
$$

and

$$
G_{\Phi^{-1}\left(S_{\varepsilon}\right)}^{\Omega_{1}}(z) \leq g_{R_{1}^{-1}}\left(\frac{1}{R_{1}} \Phi(z)\right)+1 \leq G\left(\frac{1}{R_{1}} \Phi(z)\right)+C_{3} \leq G(\Phi(z))+C_{4} .
$$

So the hypotheses of Lemma 4.5 are satisfied, with the function $G \circ \Phi$ instead of $G$.

6.4.3. Perturbations. The following lemma and its corollary will be useful to get rid of higher order perturbations of the family $\left(S_{\varepsilon}\right)$.

Lemma 6.5. Let $\Omega$ be a bounded hyperconvex domain such that the function $G_{0}^{\Omega}$ is Lipschitz in a neighborhood of $\partial \Omega$. There exists $\delta_{0}>0$ such that the following holds for any $\delta \in\left(0, \delta_{0}\right]$.

Let $S, S^{\prime} \subset B(0, \delta) \subset \subset \Omega, \# S=\# S^{\prime}=N$. Suppose there exists a biholomorphic map $\Phi$ from $\Omega$ to $\Phi(\Omega)$ such that $\Phi(S)=S^{\prime}$, and $\|\Phi(z)-z\| \leq \delta$ for any $z \in \Omega$ such that $\operatorname{dist}(z, \partial \Omega) \geq \delta$.

Then there are constants $C_{1}, C_{2}>0$ depending only on $N$ and $\Omega$ such that for any $z$ verifying $G_{\{0\}}^{\Omega}(z) \leq-C_{1} \delta$,

$$
G_{S^{\prime}}^{\Omega}(\Phi(z)) \leq G_{S}^{\Omega}(z)+C_{2} \delta .
$$

Proof. Let

$$
\Omega_{\delta}:=\left\{z \in \Omega: G_{0}^{\Omega}(z)<\inf _{\operatorname{dist}(z, \partial \Omega) \leq \delta} G_{0}^{\Omega}\right\} .
$$

Since $\Omega_{\delta} \subset\{z \in \Omega: \operatorname{dist}(z, \partial \Omega) \geq \delta\}, \Phi\left(\Omega_{\delta}\right) \subset \Omega$.

Since $G_{0}^{\Omega}$ is Lipschitz in a neighborhood of $\partial \Omega, \Omega_{\delta} \supset\{z \in \Omega$ : $\left.G_{0}^{\Omega}(z) \leq-C_{1} \delta\right\}$.

By Lemma 4.1, for $\delta$ small enough, on $\partial \Omega_{\delta}$

$$
2 N C_{1} \delta+G_{S}^{\Omega}(z) \geq-2 N G_{0}^{\Omega}(z)+G_{S}^{\Omega}(z) \geq 0 .
$$


So the plurisubharmonic functions $G_{S^{\prime}}^{\Omega} \circ \Phi$ and $G_{S}^{\Omega}+2 N C_{1} \delta$ have the same singularities on the set $S$, the first one is negative and the second one positive on $\partial \Omega_{\delta}$, and the second one is maximal psh; by [24, Lemma 4.1], we have

$$
G_{S^{\prime}}^{\Omega} \circ \Phi(z) \leq G_{S}^{\Omega}(z)+2 N C_{1} \delta
$$

for all $z \in \Omega_{\delta}$, q.e.d.

Corollary 6.6. Under the hypotheses of Lemma 6.5, suppose that we have a family $\left(S_{\varepsilon}\right)$ such that $\lim _{\varepsilon \rightarrow 0} G_{S_{\varepsilon}}(z)=g(z)$, uniformly on compacta of $\Omega \backslash\{0\}$, and a family of biholomorphic mappings $\Phi_{\varepsilon}(z)=$ $z+\Theta_{\varepsilon}(z)$, with $\lim _{\varepsilon \rightarrow 0}\left\|\Theta_{\varepsilon}\right\|_{L}=0$, where $\|\cdot\|_{L}$ denote the Lipschitz norm $\left(\|F\|_{L}:=\sup _{z \in \Omega}\|F(z)\|+\sup _{z, w \in \Omega} \frac{\|F(z)-F(w)\|}{\|z-w\|}\right)$.

Then $\lim _{\varepsilon \rightarrow 0} G_{\Phi_{\varepsilon}\left(S_{\varepsilon}\right)}(z)=g(z)$, uniformly on compacta of $\Omega \backslash\{0\}$.

Proof. Given $K \subset \subset \Omega \backslash\{0\}$ and $\eta>0$, choose $K_{1}$ such that $K \subset \subset$ $K_{1} \subset \subset \Omega \backslash\{0\}$. For $\varepsilon$ small enough, $\Phi_{\varepsilon}(K) \subset K_{1}$ and $\Phi_{\varepsilon}\left(K_{1}\right) \supset K$.

Choose $\varepsilon$ small enough so that for all $z \in K_{1},\left|G_{S_{\varepsilon}}(z)-g(z)\right|<\eta / 4$. By Lemma 6.5 applied to $\Phi_{\varepsilon}$ and to $\Phi_{\varepsilon}^{-1}$, for $\varepsilon$ small enough,

$$
G_{S_{\varepsilon}}\left(\Phi_{\varepsilon}^{-1}(z)\right)-\eta / 4 \leq G_{\Phi_{\varepsilon}\left(S_{\varepsilon}\right)}(z) \leq G_{S_{\varepsilon}}\left(\Phi_{\varepsilon}^{-1}(z)\right)+\eta / 4 .
$$

Because of the continuity of each $G_{\varepsilon}$ [18] and of the uniform convergence, $g$ is (uniformly) continuous on $K_{1}$, so that for $\varepsilon$ small enough, $z \in K_{1},\left|g(z)-g\left(\Phi_{\varepsilon}^{-1}(z)\right)\right|<\eta / 4$. Putting all the inequalities together we get $\left|G_{\Phi_{\varepsilon}\left(S_{\varepsilon}\right)}(z)-g(z)\right|<\eta$ for $z \in K$.

6.4.4. Reduction to the Model Cases. Proof of Theorem 1.12, part (1).

By applying Corollary 6.6 to the translations $\Phi_{\varepsilon}(z)=z-a_{1}^{\varepsilon}$, we see that we may assume $a_{1}^{\varepsilon}=0$ for all $\varepsilon$.

We need to check that we may apply Lemma 6.4.

Lemma 6.7. The functions $H$ and $F$ defined after Proposition 6.2 are admissible.

Proof. When there is an $i \in\{0,1,2,3\}$ such that both $z$ and $R z \in D_{i}$, the property follows immediately from the formula for $H$. Otherwise, in cases where (for instance) $z \in D_{1}$ and $R z \in D_{0}$, it is a straighforward computation. The constants $C(R)$ are fixed multiples of $\log R$. The computation is similar (if anything, easier) for $F$.

In the generic case where all the $v_{i}$ in the statement of Theorem 1.12 are distinct, we choose an invertible linear map $\Phi$ such that $\left[\Phi\left(\tilde{v}_{3}\right)\right]=$ $\left[\begin{array}{ll}1 & :\end{array}\right],\left[\Phi\left(\tilde{v}_{2}\right)\right]=[0: 1],\left[\Phi\left(\tilde{v}_{1}\right)\right]=[1:-1]$ (this follows from an elementary computation with matrices, or from the well-known fact that Möbius maps on the Riemann sphere are transitive on triples of 
points). By Lemma 6.4, we can reduce ourselves to studying $\Phi\left(S_{\varepsilon}\right)$, which means that we take

$$
a_{1}^{\varepsilon}=(0,0), \quad a_{2}^{\varepsilon}=\left(\rho_{2}(\varepsilon), \eta_{2}(\varepsilon)\right), \quad a_{3}^{\varepsilon}=\left(\eta_{3}(\varepsilon), \rho_{3}(\varepsilon)\right),
$$

with all coordinates tending to 0 and, given the new values of $v_{3}$ and $v_{2}, \lim _{\varepsilon \rightarrow 0} \eta_{j}(\varepsilon) / \rho_{j}(\varepsilon)=0, j=2,3$.

If we set $\gamma(\varepsilon):=\frac{\eta_{3}(\varepsilon)-\rho_{2}(\varepsilon)}{\rho_{3}(\varepsilon)-\eta_{2}(\varepsilon)}$, then $v_{1}^{\varepsilon}=[\gamma(\varepsilon): 1]$, so $\lim _{\varepsilon \rightarrow 0} \gamma(\varepsilon)=-1$. Hence

$$
\lim _{\varepsilon \rightarrow 0} \frac{\rho_{2}(\varepsilon)}{\rho_{3}(\varepsilon)}=\lim _{\varepsilon \rightarrow 0} \frac{\frac{\eta_{3}(\varepsilon)}{\rho_{3}(\varepsilon)}-\gamma(\varepsilon)}{1-\gamma(\varepsilon) \frac{\eta_{2}(\varepsilon)}{\rho_{2}(\varepsilon)}}=1 .
$$

Now denote by $\varepsilon$ what was denoted $\rho_{2}(\varepsilon)$ (if the application was not one-to-one, there is some ambiguity in our new notations, but all possibles choices will give subfamilies converging to the same limit). Then $\eta_{2}(\varepsilon)$ becomes $\delta_{1}(\varepsilon), \eta_{3}(\varepsilon)$ becomes $\delta_{2}(\varepsilon)$, and $\rho_{3}(\varepsilon)$ becomes $\varepsilon(1+o(1))=\varepsilon+\delta_{3}(\varepsilon)$. We are reduced to case (1) of Proposition 6.2 .

On the other hand, in the degenerate case where $v_{1}=v_{3} \neq v_{2}$, we can still take $\Phi$ such that $\left[\Phi\left(\tilde{v}_{3}\right)\right]=[1: 0]=\left[\Phi\left(\tilde{v}_{1}\right)\right],\left[\Phi\left(\tilde{v}_{2}\right)\right]=[0: 1]$. Then (6.4) and the limits following it still hold. But, setting $\gamma(\varepsilon)$ as before, we have $\lim _{\varepsilon \rightarrow 0} \gamma(\varepsilon)=\infty$, so

$$
\lim _{\varepsilon \rightarrow 0} \frac{\rho_{2}(\varepsilon)}{\rho_{3}(\varepsilon)}=\lim _{\varepsilon \rightarrow 0} \frac{\gamma(\varepsilon)^{-1} \frac{\eta_{3}(\varepsilon)}{\rho_{3}(\varepsilon)}-1}{\gamma(\varepsilon)^{-1}-\frac{\eta_{2}(\varepsilon)}{\rho_{2}(\varepsilon)}}=\infty .
$$

Again, denote by $\varepsilon$ what was denoted $\rho_{2}(\varepsilon)$, then $\rho_{3}(\varepsilon)=o\left(\rho_{2}(\varepsilon)\right)$ becomes $\delta_{4}(\varepsilon)$ and $\eta_{3}(\varepsilon)=o\left(\rho_{3}(\varepsilon)\right)$ becomes $\delta_{4}(\varepsilon) \omega(\varepsilon)$; we are reduced to case (2) of Proposition 6.2.

The properties about the decrease of $g$ in Theorem 1.12 are invariant under linear changes of coordinates, and are easily verified on the functions $F$ and $H$.

\subsection{Simplified model cases. Proof of Proposition 6.2.}

We apply Corollary 6.6 with the map

$$
\Phi_{\varepsilon}(z)=\left(z_{1}, z_{2}\right)+\left(\frac{\delta_{2}(\varepsilon)}{\varepsilon} z_{2}, \frac{\delta_{1}(\varepsilon)}{\varepsilon} z_{1}+\frac{\delta_{3}(\varepsilon)}{\varepsilon} z_{2}\right),
$$

which transforms the system $\{(0,0),(\varepsilon, 0),(0, \varepsilon)\}$ into the one given in case (1) of Proposition 6.2. The proof of that case reduces to:

Lemma 6.8 (Generic case). Suppose that $S_{\varepsilon}=\{(0,0),(\varepsilon, 0),(0, \varepsilon)\}$. Then $\lim _{\varepsilon \rightarrow 0} G_{\varepsilon}=g_{H}$, uniformly on compacta of $\mathbb{D}^{2} \backslash\{0\}$, and $g_{H} \sim_{0}$ $H$. 
For case (2), we use the map

$$
\Phi_{\varepsilon}(z)=\left(z_{1}, z_{2}\right)+\left(\omega(\varepsilon) z_{2}, \frac{\delta_{1}(\varepsilon)}{\varepsilon} z_{1}\right)
$$

which maps the system $S_{\varepsilon}=\left\{(0,0),(\varepsilon, 0),\left(0, \delta_{4}(\varepsilon)\right)\right\}$ to the one given in case $(2)$. We exchange the axes, setting $\breve{F}\left(z_{1}, z_{2}\right)=F\left(z_{2}, z_{1}\right)$. For simplicity of notation, we also write $\rho:=\delta_{4}$. The proof reduces to:

Lemma 6.9 (Degenerate case). Let $S_{\varepsilon}=\{(0,0),(\rho(\varepsilon), 0),(0, \varepsilon)\}$, where $\lim _{\varepsilon \rightarrow 0} \rho(\varepsilon) / \varepsilon=0$. Then $\lim _{\varepsilon \rightarrow 0} G_{\varepsilon}=g_{F}$, uniformly on compacta of $\mathbb{D}^{2} \backslash\{0\}$, and $g_{F} \sim_{0} \check{F}$.

6.6. Lower estimate. Now we start the proof of Lemmas 6.8 and 6.9,

Let $\psi_{\varepsilon}(z):=z_{1}+\frac{\rho(\varepsilon)}{\varepsilon} z_{2}-\rho(\varepsilon)($ where $\rho(\varepsilon)=\varepsilon$ or $\rho(\varepsilon)=o(\varepsilon))$. Let

$L_{\varepsilon}(z)=\max \left(\frac{1}{2} \log \left|z_{1} z_{2} \psi_{\varepsilon}(z)\right|, \log \left|z_{1} \frac{\rho(\varepsilon)-z_{1}}{1-\overline{\rho(\varepsilon)} z_{1}}\right|, \log \left|z_{2} \frac{\varepsilon-z_{2}}{1-\bar{\varepsilon} z_{2}}\right|\right)-\frac{1}{2} \log 3$.

Lemma 6.10. Suppose that $|\rho(\varepsilon)| \leq|\varepsilon|$ for any $\varepsilon$, then

$$
G_{\varepsilon}(z) \geq L_{\varepsilon}(z) \text {, for any } z \in \mathbb{D}^{2} .
$$

Furthermore, $\lim _{\varepsilon \rightarrow 0} L_{\varepsilon}$ exists uniformly on any compact subset of $\mathbb{D}^{2} \backslash$ $\{0\}$, and

(1) if $\rho(\varepsilon)=\varepsilon$, then $\lim _{\varepsilon \rightarrow 0} L_{\varepsilon} \sim_{0} H$;

(2) if $\rho(\varepsilon)=o(\varepsilon)$, then $\lim _{\varepsilon \rightarrow 0} L_{\varepsilon} \sim_{0} \check{F}$.

Proof. Let $S_{\varepsilon}^{\prime}:=S_{\varepsilon} \cup\{(\rho(\varepsilon), \varepsilon)\}$. This is a product set and by Lemma 6.1, we get

$$
G_{\varepsilon}(z) \geq G_{S_{\varepsilon}^{\prime}}(z)=\max \left(\log \left|z_{1} \frac{\rho(\varepsilon)-z_{1}}{1-\overline{\rho(\varepsilon)} z_{1}}\right|, \log \left|z_{2} \frac{\varepsilon-z_{2}}{1-\bar{\varepsilon} z_{2}}\right|\right) .
$$

At each of the three points of $S_{\varepsilon}$, exactly two of the three holomorphic functions $z_{1}, z_{2}$ and $\psi_{\varepsilon}(z)$ vanish, and $\left|z_{1} z_{2} \psi_{\varepsilon}(z)\right| \leq 3$ for all $z \in \mathbb{D}^{2}$, so

$$
G_{\varepsilon}(z) \geq \frac{1}{2} \log \left|z_{1} z_{2} \psi_{\varepsilon}(z)\right|-\frac{1}{2} \log 3,
$$

because the right hand side is a negative plurisubharmonic function with the correct singularities.

It is easy to see that

$$
\lim _{\varepsilon \rightarrow 0} L_{\varepsilon}(z)=\max \left(\frac{1}{2} \log \left|z_{1} z_{2}\left(z_{1}+z_{2}\right)\right|, 2 \log \left|z_{1}\right|, 2 \log \left|z_{2}\right|\right)-\frac{1}{2} \log 3
$$


if $\rho(\varepsilon)=\varepsilon$, and

$$
\lim _{\varepsilon \rightarrow 0} L_{\varepsilon}(z)=\max \left(\frac{1}{2} \log \left|z_{1}^{2} z_{2}\right|, 2 \log \left|z_{1}\right|, 2 \log \left|z_{2}\right|\right)-\frac{1}{2} \log 3
$$

if $\rho(\varepsilon)=o(\varepsilon)$, with uniform convergence on any compact subset of $\mathbb{D}^{2} \backslash\{0\}$.

We give only the estimate from below of $\lim _{\varepsilon \rightarrow 0} L_{\varepsilon}$ in the inequalities implicit in (1) and (2) (those are the only ones needed to prove Lemmas 6.8 and 6.9).

First we deal with statement (1) in the Lemma.

For $z \in D_{0}$, the inequality is immediate. For $z \in D_{1},\left|\frac{z_{2}}{z_{1}}+1\right| \geq \frac{1}{2}$, so $\left|z_{1} z_{2}\left(z_{1}+z_{2}\right)\right| \geq\left|z_{1} z_{2}\right| \frac{1}{2}\left|z_{1}\right|$, and we get

$$
\lim _{\varepsilon \rightarrow 0} L_{\varepsilon}(z) \geq \frac{1}{2} \log \left|z_{1} z_{2}\left(z_{1}+z_{2}\right)\right|-\frac{1}{2} \log 3 \geq H(z)-\frac{1}{2} \log 6 .
$$

By considering the image of the disk $\mathcal{D}_{3}$ under the inversion $\zeta \mapsto$ $1 / \zeta$, we see that $\left|\frac{z_{2}}{z_{1}}+1\right| \geq \frac{1}{2}$ implies $\left|\frac{z_{1}}{z_{2}}+1\right| \geq \frac{1}{3}$, so for $z \in D_{2}$, $\frac{1}{2} \log \left|z_{1} z_{2}\left(z_{1}+z_{2}\right)\right| \geq \frac{1}{2} \log \left|z_{1} z_{2}^{2}\right|-\frac{1}{2} \log 3=H(z)-\frac{1}{2} \log 3$.

Finally, for $z \in D_{3},\left|\frac{z_{2}}{z_{1}}+1\right| \leq \frac{1}{2}$, so $\left|z_{2}\right| \geq \frac{1}{2}\left|z_{1}\right|$ and $\frac{1}{2} \log \left|z_{1} z_{2}\left(z_{1}+z_{2}\right)\right| \geq$ $\frac{1}{2} \log \left|z_{1}^{2}\left(z_{1}+z_{2}\right)\right|-\frac{1}{2} \log 2=H(z)-\frac{1}{2} \log 2$.

In case (2), the computations are even easier.

6.7. Upper estimates for the generic case. We will now estimate $G_{\varepsilon}$ from above by constructing certain analytic discs. This should be compared with 25$]$.

In what follows, $S_{\varepsilon}$ and $G_{\varepsilon}$ are as in Lemma 6.8.

\subsubsection{Near the coordinate axes.}

Lemma 6.11. Let $\delta>0$. For any $\eta>0$ there exists $m=m(\delta, \eta)>0$ such that for any $\varepsilon \in D(0, m)$, for any $z_{1}, z_{2} \in D_{0} \backslash D(0, \delta)^{2}$,

$$
G_{\varepsilon}\left(z_{1}, z_{2}\right) \leq 2 \log \left(\max \left(\left|z_{1}\right|,\left|z_{2}\right|\right)\right)+\eta .
$$

Proof. Suppose for instance that $\left|z_{2}\right| \leq\left|z_{1}\right|^{2}$. Then $\max \left(\left|z_{1}\right|,\left|z_{2}\right|\right)=$ $\left|z_{1}\right|$ and $\delta \leq\left|z_{1}\right|$. Let

$$
\tilde{\varphi}_{\varepsilon}(\zeta):=\left(\zeta, \frac{\zeta(\zeta-\varepsilon)}{z_{1}\left(z_{1}-\varepsilon\right)} z_{2}\right) .
$$

Since

$$
\left|\frac{z_{2}}{z_{1}\left(z_{1}-\varepsilon\right)}\right|=\left|\frac{z_{2}}{z_{1}^{2}} \frac{1}{1-\frac{\varepsilon}{z_{1}}}\right| \leq \frac{1}{\left|1-\frac{|\varepsilon|}{\delta}\right|}
$$


we can choose $0<\gamma=O(|\varepsilon|)$ such that $\varphi(\zeta):=\tilde{\varphi}\left(\frac{\zeta}{1+\gamma}\right)$ defines a map from $\mathbb{D}$ to $\mathbb{D}^{2}$.

Now $G_{\varepsilon} \circ \varphi \in S H_{-}(\mathbb{D})$, and it has logarithmic singularities at 0 and $(1+\gamma) \varepsilon$, so (using the explicit formula for the Green function in the unit disk)

$$
G_{\varepsilon}\left(z_{1}, z_{2}\right)=G_{\varepsilon} \circ \varphi\left((1+\gamma) z_{1}\right) \leq \log \left|(1+\gamma) z_{1} \frac{(1+\gamma)\left(z_{1}-\varepsilon\right)}{1-|1+\gamma|^{2} z_{1} \bar{\varepsilon}}\right|,
$$

which yields the required inequality for $|\varepsilon|<m$.

If $\left|z_{1}\right| \leq\left|z_{2}\right|^{2}$, we just exchange the roles of the coordinates. If $\left|z_{1}+z_{2}\right| \leq\left|z_{1}\right|^{2}$, we perform an analogous computation with

$$
\tilde{\varphi}_{\varepsilon}(\zeta):=(\zeta,(\zeta-\varepsilon)(\alpha \zeta-1)), \quad \alpha:=\frac{z_{1}+z_{2}-\varepsilon}{z_{1}\left(z_{1}-\varepsilon\right)} .
$$

6.7.2. Analytic discs for the most common case. Away from the exceptional region $D_{0}$, the construction of the analytic disks is more delicate.

Lemma 6.12. Let $\delta>0$. Let $\left(z_{1}, z_{2}\right) \in \mathbb{D}^{2} \backslash\left(D_{0} \cup D(0, \delta)^{2}\right),\left|\frac{z_{1}}{z_{2}}+1\right| \geq$ $\frac{1}{2}$.

For any $\eta>0$ there exists $m>0$ such that for any $\alpha \in D(0, m)$, there exists $\varphi \in \mathcal{O}\left(\mathbb{D}, \mathbb{D}^{2}\right)$ and $\zeta_{1}, \zeta_{2} \in D(0, \eta), \zeta_{3} \in \mathbb{D}$ such that $\left.|| \zeta_{3}\right|^{2}-\max \left(\left|z_{1}\right|,\left|z_{2}\right|\right) \mid \leq \eta$ and

$$
\varphi(0)=(0,0), \varphi\left(\zeta_{1}\right)=\left(\alpha^{2}, 0\right), \varphi\left(\zeta_{2}\right)=\left(0, \alpha^{2}\right), \varphi\left(\zeta_{3}\right)=\left(z_{1}, z_{2}\right) .
$$

Proof. Case 1. $\left|z_{2}\right|^{2} \leq\left|z_{1}\right| \leq\left|z_{2}\right|$.

Let $\xi_{1}, \xi_{2} \in D\left(0, \frac{1}{2}\right) \subset \mathbb{C}$. We define a mapping from $\mathbb{D}$ to $\mathbb{C}^{2}$ by

$$
\tilde{\varphi}_{\xi}(\zeta):=\left(\frac{z_{1}}{z_{2}}\left(1+\xi_{1}\right) \zeta\left(\zeta-\tilde{\zeta}_{2}\right),\left(1+\xi_{2}\right) \zeta\left(\zeta-\tilde{\zeta}_{1}\right)\right)
$$

with $\tilde{\zeta}_{1}, \tilde{\zeta}_{2}$ to be defined below. We note that $\tilde{\varphi}_{\xi}(0)=(0,0)$, and

$$
\begin{aligned}
& \tilde{\varphi}_{\xi}\left(\tilde{\zeta}_{1}\right)=\left(\frac{z_{1}}{z_{2}}\left(1+\xi_{1}\right) \tilde{\zeta}_{1}\left(\tilde{\zeta}_{1}-\tilde{\zeta}_{2}\right), 0\right), \\
& \tilde{\varphi}_{\xi}\left(\tilde{\zeta}_{2}\right)=\left(0,\left(1+\xi_{2}\right) \tilde{\zeta}_{2}\left(\tilde{\zeta}_{2}-\tilde{\zeta}_{1}\right)\right) .
\end{aligned}
$$

We will need the auxiliary quantity

$$
\mu:=\alpha\left(\frac{1}{1+\xi_{2}}+\frac{1}{1+\xi_{1}} \frac{z_{2}}{z_{1}}\right)^{1 / 2},
$$


where we choose one particular square root of $1+\frac{z_{2}}{z_{1}}$, and define the above square root to be continuous in a neighborhood of $\xi_{1}=0, \xi_{2}=0$ (we may need to reduce the size of the disk where $\xi_{1}, \xi_{2}$ can be chosen).

Note that $\left|\frac{z_{1}}{z_{2}}+1\right| \geq \frac{1}{2}$ implies $\left|\frac{z_{2}}{z_{1}}+1\right| \geq \frac{1}{3}$, and that $\left|\frac{z_{2}}{z_{1}}\right|=\left|\frac{z_{2}^{2}}{z_{1}} \frac{1}{z_{2}}\right| \leq$ $\frac{1}{\delta}$, so that for $\xi_{1}, \xi_{2}$ small enough,

$$
\frac{1}{4}|\alpha| \leq|\mu| \leq \frac{2}{\sqrt{\delta}}|\alpha| \text {. }
$$

We now set

$$
\tilde{\zeta}_{1}:=\frac{\alpha^{2}}{\left(1+\xi_{1}\right) \mu} \frac{z_{2}}{z_{1}}, \quad \tilde{\zeta}_{2}:=\tilde{\zeta}_{1}-\mu=-\frac{\alpha^{2}}{\left(1+\xi_{2}\right) \mu} .
$$

Substituting this into (6.6), (6.7), we get

$$
\begin{aligned}
& \tilde{\varphi}_{\xi}\left(\tilde{\zeta}_{1}\right)=\left(\frac{z_{1}}{z_{2}}\left(1+\xi_{1}\right) \tilde{\zeta}_{1} \mu, 0\right)=\left(\alpha^{2}, 0\right), \\
& \tilde{\varphi}_{\xi}\left(\tilde{\zeta}_{2}\right)=\left(0,-\left(1+\xi_{2}\right) \tilde{\zeta}_{2} \mu\right)=\left(0, \alpha^{2}\right) .
\end{aligned}
$$

We have an analytic disk passing through all three poles, now we need to have it go through the point $z$. Let $\tilde{\zeta}_{3}:=z_{2}^{1 / 2}$ (it doesn't matter which square root we choose). Then

$$
\begin{array}{r}
\tilde{\varphi}_{\xi}\left(\tilde{\zeta}_{3}\right)-\left(z_{1}, z_{2}\right)=\left(z_{1} \xi_{1}\right. \\
\left.+\frac{\left(1+\xi_{1}\right) \alpha^{2}}{\left(1+\xi_{2}\right) \mu} \frac{z_{1}}{z_{2}^{1 / 2}}, z_{2} \xi_{2}-\frac{\left(1+\xi_{2}\right) \alpha^{2}}{\left(1+\xi_{1}\right) \mu} \frac{z_{2}^{3 / 2}}{z_{1}}\right) \\
=:\left(z_{1}\left(\xi_{1}-\Phi_{1}(\xi, \alpha)\right), z_{2}\left(\xi_{2}-\Phi_{2}(\xi, \alpha)\right)\right) .
\end{array}
$$

Since $\alpha^{2} / \mu=O(|\alpha|)$ and $\left|\frac{z_{1}}{z_{2}^{1 / 2}}\right| \leq 1 / \eta,\left|\frac{z_{2}^{3 / 2}}{z_{1}}\right| \leq 1 / \eta^{2}$, for $|\alpha|$ small enough, the map $\xi \mapsto \Phi(\xi, \alpha)$ is contractive, so there exists a unique $\xi^{0}$ such that $\xi^{0}=\Phi\left(\xi^{0}, \alpha\right)$, and $\left\|\xi^{0}\right\| \leq C \delta^{-1}|\alpha|$. Let $\tilde{\varphi}(\zeta):=\tilde{\varphi}_{\xi^{0}}(\zeta)$.

The map $\tilde{\varphi}$ hits the correct points, but in general $\tilde{\varphi}(\mathbb{D}) \not \subset \mathbb{D}^{2}$. However, the estimates on $\mu$ and $\xi^{0}$ imply that

$$
\tilde{\varphi}(\zeta)=\left(\frac{z_{1}}{z_{2}} \zeta^{2}, \zeta^{2}\right)+O\left(\delta^{-1}|\alpha|\right),
$$

so using the Schwarz Lemma there exists $0<\gamma=O\left(\delta^{-1}|\alpha|\right)$ such that letting

$$
\varphi(\zeta):=\tilde{\varphi}\left(\frac{\zeta}{1+\gamma}\right), \quad \zeta_{j}:=(1+\gamma) \tilde{\zeta}_{j}, 1 \leq j \leq 3,
$$

we have a map and points in the disk satisfying the conclusions of the Lemma for $|\alpha|$ small enough.

Case 2. $\left|z_{1}\right|^{2} \leq\left|z_{2}\right| \leq\left|z_{1}\right|$. 
The computations are almost exactly the same once we exchange $z_{1}$ and $z_{2}$.

6.7.3. Upper estimate from the analytic disks. It is easy to deduce from the previous Lemma a rough global estimate on the function $G_{\varepsilon}$.

Lemma 6.13. Then there exists $C>0$ such that for any $\delta>0$, there is an $m=m(\delta)>0$ such that for any $\varepsilon \in D(0, m)$, for any $\left(z_{1}, z_{2}\right) \in \mathbb{D}^{2} \backslash\left(D_{0} \cup D(0, \delta)^{2}\right)$,

$$
G_{\varepsilon}\left(z_{1}, z_{2}\right) \leq \frac{3}{2} \log \left(\max \left(\left|z_{1}\right|,\left|z_{2}\right|\right)\right)+C .
$$

Notice that for $\left(z_{1}, z_{2}\right) \in D_{0} \backslash D(0, \delta)^{2}$, Lemma 6.11 gives an even better estimate, so that the result actually holds on the whole of $\mathbb{D}^{2} \backslash$ $D(0, \delta)^{2}$.

Proof. If $\left|\frac{z_{1}}{z_{2}}+1\right| \geq \frac{1}{2}$, then by choosing an analytic disk $\varphi$ as in Lemma 6.12 with $\alpha=\varepsilon^{1 / 2}$, we have $G_{\varepsilon} \circ \varphi \in S H_{-}(\mathbb{D})$, with three logarithmic poles, and a reasoning similar to the proof of Lemma 6.11 shows that $G_{\varepsilon}(z) \leq \frac{3}{2} \log \left(\max \left(\left|z_{1}\right|,\left|z_{2}\right|\right)\right)+1$ when $\eta$ is small enough, and therefore when $|\varepsilon|$ is small enough.

To deal with the remaining case, consider the affine biholomorphism

$$
L\left(z_{1}, z_{2}\right):=\left(z_{1}, \varepsilon-z_{1}-z_{2}\right) .
$$

It sends $S_{3, \varepsilon}$ to itself (changing the roles of the points) and the set

$$
\begin{aligned}
& \left\{\left|\frac{z_{1}}{z_{2}}+1\right|<\frac{1}{2}\right\} \text { to }\left\{\left|\frac{w_{1}}{w_{2}-\varepsilon}+1\right|>2\right\} \text {. Also, } L^{-1}\left(\mathbb{D}^{2}\right) \subset 3 \mathbb{D}^{2} \text { for }|\varepsilon| \leq 1 . \\
& \text { So } \\
& \qquad G_{\varepsilon}^{\mathbb{D}^{2}}\left(\frac{z}{3}\right)=G_{3 \varepsilon}^{3 \mathbb{D}^{2}}(z) \leq G_{3 \varepsilon}^{L^{-1}\left(\mathbb{D}^{2}\right)}(z)=G_{3 \varepsilon}^{\mathbb{D}^{2}}(L(z)),
\end{aligned}
$$

therefore for $|\varepsilon|$ small enough with respect to $\delta$, we have $\left|\frac{L_{1}(3 z)}{L_{2}(3 z)}+1\right|>\frac{1}{2}$ and

$$
\begin{aligned}
& G_{\varepsilon}^{\mathbb{D}^{2}}(z) \leq G_{3 \varepsilon}^{\mathbb{D}^{2}}(L(3 z)) \\
& \leq \frac{3}{2} \log \left(\max \left(\left|L_{1}(3 z)\right|,\left|L_{2}(3 z)\right|\right)\right)+1 \leq \frac{3}{2} \log \left(\max \left(\left|z_{1}\right|,\left|z_{2}\right|\right)\right)+C .
\end{aligned}
$$

\subsubsection{End of proof of Lemma 6.8.}

Any compact subset of $\mathbb{D}^{2} \backslash\{0\}$ is contained in $\mathbb{D}^{2} \backslash D(0, \delta)^{2}$ for some $\delta>0$.

Case 1. $\left|z_{1}\right|^{2} \leq\left|z_{2}\right| \leq\left|z_{1}\right|$.

Consider the holomorphic map from $\mathbb{D}$ to $\mathbb{D}^{2}$ given by $\varphi(\zeta)=\left(\zeta, \frac{z_{2}}{z_{1}} \zeta\right)$, and $u:=G_{\varepsilon} \circ \varphi \in S H_{-}(\mathbb{D})$. By Lemma 6.13, for any $\delta_{1}>0$ and $\zeta$ such 
that $|\zeta|=\delta_{1},|\varepsilon|<m\left(\delta_{1}\right)$, we have $u(\zeta) \leq \frac{3}{2} \log \delta_{1}+C$. On the other hand, when $\left|z_{2}\right|<\left|z_{1}\right|$, if $|\zeta|=\left|\frac{z_{2}}{z_{1}}\right|$, then $\varphi(\zeta) \in\left\{w:\left|w_{2}\right| \leq\left|w_{1}\right|^{2}\right\}$ and by Lemma 6.11, for any $\eta_{1}>0$ we can choose $|\varepsilon|$ small enough so that

$$
u(\zeta) \leq 2 \log \left|\frac{z_{2}}{z_{1}}\right|+\eta_{1}
$$

Applying the three-circle theorem, we get, for any $\zeta \in D\left(0,\left|\frac{z_{2}}{z_{1}}\right|\right) \backslash$ $D\left(0, \delta_{1}\right)$,

$$
u(\zeta) \leq \frac{\log \left|\frac{z_{2}}{z_{1}}\right|-\log |\zeta|}{\log \left|\frac{z_{2}}{z_{1}}\right|-\log \delta_{1}}\left(\frac{3}{2} \log \delta_{1}+C\right)+\frac{\log |\zeta|-\log \delta_{1}}{\log \left|\frac{z_{2}}{z_{1}}\right|-\log \delta_{1}}\left(2 \log \left|\frac{z_{2}}{z_{1}}\right|+\eta_{1}\right) .
$$

This inequality still holds in the case $\left|z_{2}\right|=\left|z_{1}\right|$, simply using the fact that $u \leq 0$. We may apply it for $\zeta=z_{1}$. Let $\eta_{2}>0$. Then

$$
\frac{\log \left|\frac{z_{2}}{z_{1}}\right|-\log \left|z_{1}\right|}{\log \left|\frac{z_{2}}{z_{1}}\right|-\log \delta_{1}}\left(\frac{3}{2} \log \delta_{1}+C\right)=\left(\log \left|z_{2}\right|-2 \log \left|z_{1}\right|\right) \frac{-\frac{3}{2}+\frac{C}{\left|\log \delta_{1}\right|}}{1-\frac{|\log | \frac{z_{2}}{z_{1}} \mid}{\left|\log \delta_{1}\right|}},
$$

and for $\delta_{1}$ small enough (depending on $\eta_{2}$ and $\delta$ ),

$$
\frac{-\frac{3}{2}+\frac{C}{\left|\log \delta_{1}\right|}}{1-\frac{|\log | \frac{z_{2}}{z_{1}} \mid}{\left|\log \delta_{1}\right|}} \leq-\frac{3}{2}+\frac{C}{\left|\log \delta_{1}\right|} \leq-\frac{3}{2}+\frac{\eta_{2}}{|\log \delta|} \leq-\frac{3}{2}+\frac{\eta_{2}}{\log \left|z_{2}\right|-2 \log \left|z_{1}\right|},
$$

since $0<\log \left|z_{2}\right|-2 \log \left|z_{1}\right| \leq-\log \left|z_{1}\right| \leq-\log \delta$. Finally the first term of the right hand side of (6.10) is bounded above by

$$
-\frac{3}{2}\left(\log \left|z_{2}\right|-2 \log \left|z_{1}\right|\right)+\eta_{2}
$$

For the second term, note first that if $\left(2 \log \left|\frac{z_{2}}{z_{1}}\right|+\eta_{1}\right)>0$, we can just bound the second term by that quantity. Otherwise, for $\left|z_{2}\right|<\left|z_{1}\right|$ and $\delta_{1}$ small enough (depending only on $\delta$ )

$$
\begin{aligned}
& \frac{\log \left|z_{1}\right|-\log \delta_{1}}{\log \left|\frac{z_{2}}{z_{1}}\right|-\log \delta_{1}}=\frac{1-\frac{|\log | z_{1}||}{\left|\log \delta_{1}\right|}}{1-\frac{|\log | \frac{z_{2}}{z_{1}} \mid}{\left|\log \delta_{1}\right|}} \geq 1-\frac{|\log | z_{1}||}{\left|\log \delta_{1}\right|} \geq \\
& 1-\frac{\eta_{2}}{2|\log \delta|} \geq 1-\frac{\eta_{2}}{2|\log | \frac{z_{2}}{z_{1}}||} \geq 1+\frac{\eta_{2}}{2 \log \left|\frac{z_{2}}{z_{1}}\right|+\eta_{1}}
\end{aligned}
$$


and therefore the second term verifies

$$
\frac{\log \left|z_{1}\right|-\log \delta_{1}}{\log \left|\frac{z_{2}}{z_{1}}\right|-\log \delta_{1}}\left(2 \log \left|\frac{z_{2}}{z_{1}}\right|+\eta_{1}\right) \leq 2 \log \left|\frac{z_{2}}{z_{1}}\right|+\eta_{1}+\eta_{2} .
$$

For $\left|z_{1}\right|=\left|z_{2}\right|$, this inequality is immediate Putting together the two estimates, for $\delta_{1}$ small enough (depending on $\delta, \eta_{2}$ ), and for $|\varepsilon|$ small enough (depending on $\delta_{1}, \eta_{1}$ ),

$$
G_{\varepsilon}\left(z_{1}, z_{2}\right)=u\left(z_{1}\right) \leq \log \left|z_{1}\right|+\frac{1}{2} \log \left|z_{2}\right|+\eta_{1}+2 \eta_{2}
$$

which yields the upper estimate from Lemma 6.8.

Case 2. $\left|z_{2}\right|^{2} \leq\left|z_{1}\right| \leq\left|z_{2}\right|$.

The same proof works, exchanging the roles of the coordinates.

Case 3. $\left|z_{1}\right|^{2} \leq\left|z_{1}+z_{2}\right| \leq \frac{1}{2}\left|z_{1}\right|$.

Using the same map $\varphi$, defined only for $|\zeta|<2 / 3$, computations similar to those of Case 1 (where $z_{2}$ is replaced by $z_{1}+z_{2}$ ) yield the desired result.

6.8. Upper estimates for the degenerate case. From now on, $S_{\varepsilon}$ and $G_{\varepsilon}$ are as in Lemma 6.9.

\subsubsection{Upper estimate for the most common case.}

Lemma 6.14. Let $\delta>0$. Let $\left(z_{1}, z_{2}\right) \in \mathbb{D}^{2} \backslash\left(D_{0} \cup D(0, \delta)^{2}\right)$. Let $s$ be a function in a neighborhood of 0 is $\mathbb{C}$ such that $\lim _{\zeta \rightarrow 0} s(\zeta)=0$.

Then there exists $m>0$ such that for any $\alpha \in D(0, m)$, and for any $\eta>0$ there exists $\varphi \in \mathcal{O}\left(\mathbb{D}, \mathbb{D}^{2}\right)$ and $\zeta_{1}, \zeta_{2} \in D(0, \eta), \zeta_{3} \in \mathbb{D}$ such that $\left.|| \zeta_{3}\right|^{2}-\max \left(\left|z_{1}\right|,\left|z_{2}\right|\right) \mid \leq \eta$ and

$$
\varphi(0)=(0,0), \varphi\left(\zeta_{1}\right)=\left([\alpha s(\alpha)]^{2}, 0\right), \varphi\left(\zeta_{2}\right)=\left(0, \alpha^{2}\right), \varphi\left(\zeta_{3}\right)=\left(z_{1}, z_{2}\right) .
$$

Proof. We proceed as in the proof of Lemma 6.12, with a few changes.

Case 1. $\left|z_{2}\right|^{2} \leq\left|z_{1}\right| \leq\left|z_{2}\right|$.

Let $\xi_{1}, \xi_{2} \in \underset{\sim}{D}\left(0, \frac{1}{2}\right) \subset \mathbb{C}$. We define the mapping $\tilde{\varphi}_{\xi}$ from $\mathbb{D}$ to $\mathbb{C}^{2}$ as before, but $\tilde{\zeta}_{1}, \tilde{\zeta}_{2}$ are defined by

$$
\begin{gathered}
\mu:=\alpha\left(\frac{1}{1+\xi_{2}}+\frac{s(\alpha)^{2}}{1+\xi_{1}} \frac{z_{2}}{z_{1}}\right)^{1 / 2}, \\
\tilde{\zeta}_{1}:=\frac{\alpha^{2} s(\alpha)^{2}}{\left(1+\xi_{1}\right) \mu} \frac{z_{2}}{z_{1}}, \quad \tilde{\zeta}_{2}:=\tilde{\zeta}_{1}-\mu=-\frac{\alpha^{2}}{\left(1+\xi_{2}\right) \mu} .
\end{gathered}
$$

This ensures that $\tilde{\varphi}_{\xi}$ takes the required values at $\tilde{\zeta}_{1}$ and $\tilde{\zeta}_{2}$. 
As before, $\left|\frac{z_{2}}{z_{1}}\right| \leq \frac{1}{\delta}$, so that for $\xi_{1}, \xi_{2}$ small enough and $|\alpha| \leq m(\delta)$, $\left|\frac{s(\alpha)^{2}}{1+\xi_{1}} \frac{z_{2}}{z_{1}}\right| \leq \frac{1}{6}$, thus

$$
\frac{1}{2}|\alpha| \leq|\mu| \leq 2|\alpha|
$$

The only point that remains to be modified in the previous proof is that

$$
\Phi_{2}(\xi, \alpha):=-\frac{\left(1+\xi_{2}\right) \alpha^{2} s(\alpha)^{2}}{\left(1+\xi_{1}\right) \mu} \frac{z_{2}^{1 / 2}}{z_{1}},
$$

which can only improve the contractivity of $\Phi$.

Case 2. $\left|z_{1}\right|^{2} \leq\left|z_{2}\right| \leq\left|z_{1}\right|$.

There we set

$$
\begin{gathered}
\mu:=\alpha\left(\frac{z_{1}}{z_{2}} \frac{1}{1+\xi_{2}}+\frac{s(\alpha)^{2}}{1+\xi_{1}}\right)^{1 / 2}, \\
\tilde{\zeta}_{1}:=-\frac{\alpha^{2} s(\alpha)^{2}}{\left(1+\xi_{1}\right) \mu}, \quad \tilde{\zeta}_{2}:=\tilde{\zeta}_{1}+\mu=-\frac{\alpha^{2}}{\left(1+\xi_{2}\right) \mu} \frac{z_{1}}{z_{2}} .
\end{gathered}
$$

Again, we see that $1 \leq\left|\frac{z_{1}}{z_{2}}\right| \leq \frac{1}{\delta}$, so for $\alpha$ small enough,

$$
\frac{1}{\sqrt{2}}|\alpha| \leq|\mu| \leq \frac{2}{\sqrt{\delta}}|\alpha| \text {. }
$$

The proof proceeds as before, with the single change

$$
\Phi_{2}(\xi, \alpha):=\frac{\left(1+\xi_{2}\right) \alpha^{2} s(\alpha)^{2}}{\left(1+\xi_{1}\right) \mu} \frac{1}{z_{1}^{1 / 2}} .
$$

The following statement is proved from the previous Lemma exactly as Lemma 6.13, with a simpler proof as we don't need to consider the values of $\frac{z_{1}}{z_{2}}+1$.

Corollary 6.15. Under the hypotheses of Lemma 6.14, there exists $C>0$ such that for any $\delta>0$, there is an $m=m(\delta)>0$ such that for any $\varepsilon \in D(0, m)$, for any $\left(z_{1}, z_{2}\right) \in \mathbb{D}^{2} \backslash\left(D_{0} \cup D(0, \delta)^{2}\right)$,

$$
G_{\varepsilon}\left(z_{1}, z_{2}\right) \leq \frac{3}{2} \log \left(\max \left(\left|z_{1}\right|,\left|z_{2}\right|\right)\right)+C .
$$


6.8.2. Upper estimate near the coordinate axes. Now we need to estimate the (faster) decrease of the Green function on the exceptional region $\check{D}_{0}^{\prime}:=\left\{z \in \mathbb{D}^{2} \backslash\{0\}:\left|z_{1}\right| \leq\left|z_{2}\right|^{3 / 2}\right.$ or $\left.\left|z_{2}\right| \leq\left|z_{1}\right|^{2}\right\}$.

Lemma 6.16. Let $\delta>0$. For any $\eta>0$ there exists $m=m(\delta, \eta)>0$ such that for any $\varepsilon \in D(0, m)$, for any $\left(z_{1}, z_{2}\right) \in \check{D}_{0}^{\prime} \backslash D(0, \delta)^{2}$,

$$
G_{\varepsilon}\left(z_{1}, z_{2}\right) \leq 2 \log \left(\max \left(\left|z_{1}\right|,\left|z_{2}\right|\right)\right)+\eta .
$$

Proof. In the cases $\left|z_{2}\right| \leq\left|z_{1}\right|^{2}$ or $\left|z_{1}\right| \leq\left|z_{2}\right|^{2}$, the proof of Lemma 6.11 will apply.

When $\left|z_{2}\right|^{2}<\left|z_{1}\right| \leq\left|z_{2}\right|^{3 / 2}$, some new trick is required. This time, the analytic disk we construct will have to pass twice through one of the poles; this should be compared to Poletsky's theorem [23], 22], see also [17], where the Green function is recovered from analytic disks that may have to hit the pole(s) more than once. Our disks will be perturbations of the Neil parabola $\zeta \mapsto\left(\zeta^{3}, \zeta^{2}\right)$.

We write $s(\varepsilon)=\rho(\varepsilon) / \varepsilon=o(1)$.

Choose complex numbers $\lambda, \mu$ such that

$$
\lambda^{2}:=\frac{z_{1}}{z_{2}\left(z_{2}-\varepsilon\right)}\left(\frac{z_{1}}{z_{2}-\varepsilon}+s(\varepsilon)\right) ; \quad \mu^{2}:=\varepsilon+\left(\frac{s(\varepsilon)}{2 \lambda}\right)^{2} .
$$

Let

$$
\Psi_{\lambda, \mu}(\zeta):=\left(\left(\lambda \zeta-\frac{1}{2} s(\varepsilon)\right)\left(\zeta^{2}-\mu^{2}\right), \zeta^{2}-\left(\frac{s(\varepsilon)}{2 \lambda}\right)^{2}\right)
$$

Then by construction

$\Psi_{\lambda, \mu}(\mu)=\Psi_{\lambda, \mu}(-\mu)=(0, \varepsilon), \Psi_{\lambda, \mu}\left(\frac{s(\varepsilon)}{2 \lambda}\right)=(0,0), \Psi_{\lambda, \mu}\left(-\frac{s(\varepsilon)}{2 \lambda}\right)=(\varepsilon s(\varepsilon), 0)$,

so we have a disk passing through all three poles of $G_{\varepsilon}$. Furthermore, choosing

$$
\zeta_{z}:=\frac{1}{\lambda}\left(\frac{z_{1}}{z_{2}-\varepsilon}+s(\varepsilon)\right)
$$

we have $\Psi_{\lambda, \mu}\left(\zeta_{z}\right)=z$. Notice that

$$
\zeta_{z}^{2}=\frac{z_{2}\left(z_{2}-\varepsilon\right)}{z_{1}}\left(\frac{z_{1}}{z_{2}-\varepsilon}+s(\varepsilon)\right),
$$

so there is some $\varepsilon_{0}(\delta, \eta)>0$ such that for $|\varepsilon|<\varepsilon_{0}(\delta, \eta)$, for any $z \notin$ $D(0, \delta)^{2}$ such that $\left|z_{2}\right|^{2}<\left|z_{1}\right| \leq\left|z_{2}\right|^{3 / 2}$,

$$
\left.|| \zeta_{z}|-| z_{2}\right|^{1 / 2} \mid \leq \eta
$$

In particular, if $z$ remains in a compact subset of $\mathbb{D}^{2}$ avoiding the origin, by choosing $\eta$ small enough we ensure that $\zeta \in \mathbb{D}$. We need a more general fact. 
Claim.

Let $\eta>0$, and $\delta>0$. Then there exists $\varepsilon_{1}=\varepsilon_{1}(\delta, \eta)>0$ such that for any $\varepsilon$ with $|\varepsilon| \leq \varepsilon_{1}$, for any $z \in \mathbb{D}^{2} \backslash D(0, \delta)^{2}$ such that $\left|z_{2}\right|^{2}<\left|z_{1}\right| \leq\left|z_{2}\right|^{3 / 2}$, we have $\Psi_{\lambda, \mu}(D(0,1-\eta)) \subset \mathbb{D}^{2}$.

Proof of the Claim.

For $|\varepsilon| \leq \delta / 2,\left|z_{2}\right| / 2 \leq\left|z_{2}-\varepsilon\right| \leq 2\left|z_{2}\right|$, so

$$
|\lambda|^{2} \geq\left|\frac{z_{1}}{2 z_{2}^{2}}\right|\left(\left|\frac{z_{1}}{2 z_{2}}\right|-|s(\varepsilon)|\right) \geq\left|\frac{z_{1}^{2}}{8 z_{2}^{3}}\right| \geq \frac{\delta}{8}
$$

for $\varepsilon$ small enough. So when $|\zeta| \leq 1-\eta$,

$$
\left|\Psi_{\lambda, \mu, 2}(\zeta)\right| \leq(1-\eta)^{2}+\frac{2|s(\varepsilon)|^{2}}{\delta}<1
$$

for $\varepsilon$ small enough.

In a similar way, given $\eta^{\prime}$, for $\varepsilon$ small enough depending on $\delta$ and $\eta^{\prime}$, we have $\left|z_{2}\right| \leq\left(1+\eta^{\prime}\right)\left|z_{2}-\varepsilon\right|$, so

$$
|\lambda|^{2} \leq\left(1+\eta^{\prime}\right)^{2}\left|\frac{z_{1}}{z_{2}^{2}}\right|\left(\left|\frac{z_{1}}{z_{2}}\right|+\frac{|s(\varepsilon)|}{\left(1+\eta^{\prime}\right)}\right) \leq\left(1+\eta^{\prime}\right)^{3}\left|\frac{z_{1}^{2}}{z_{2}^{3}}\right| \leq\left(1+\eta^{\prime}\right)^{3}
$$

for $\varepsilon$ small enough. Choose $\eta^{\prime}$ so that $\left(1+\eta^{\prime}\right)^{3}=(1+\eta)$. When $|\zeta| \leq 1-\eta$

$\left|\Psi_{\lambda, \mu, 1}(\zeta)\right| \leq\left((1+\eta)(1-\eta)+\frac{1}{2}|s(\varepsilon)|\right)\left((1-\eta)^{2}+|\varepsilon|+\frac{4|s(\varepsilon)|^{2}}{\delta}\right)<1$

for $\varepsilon$ small enough.

So now the function $v(\zeta):=G_{\varepsilon}\left(\Psi_{\lambda, \mu}((1-\eta) \zeta)\right)$ is negative and subharmonic on $\mathbb{D}$. Furthermore, it has logarithmic poles at the points $\pm \frac{\mu}{1-\eta}$ and $\pm \frac{s(\varepsilon)}{2 \lambda(1-\eta)}$; in the cases when $\mu=0$ or $s(\varepsilon)=0$, we get a double logarithmic pole at the corresponding point.

Denote $d_{\mathbb{D}}(\zeta, \xi):=\left|\frac{\zeta-\xi}{1-\zeta \xi}\right|$. Then

$$
\begin{aligned}
G_{\varepsilon}(z)=v\left(\zeta_{z}\right) \leq & \log d_{\mathbb{D}}\left(\zeta_{z}, \frac{\mu}{1-\eta}\right)+\log d_{\mathbb{D}}\left(\zeta_{z},-\frac{\mu}{1-\eta}\right) \\
& +\log d_{\mathbb{D}}\left(\zeta_{z}, \frac{s(\varepsilon)}{2 \lambda(1-\eta)}\right)+\log d_{\mathbb{D}}\left(\zeta_{z},-\frac{s(\varepsilon)}{2 \lambda(1-\eta)}\right)
\end{aligned}
$$

By (6.11), choosing $m(\delta, \eta)$ accordingly, we have, for $|\varepsilon| \leq m, G_{\varepsilon}(z) \leq$ $4 \log \left|z_{2}\right|^{1 / 2}+O(\eta)$. Changing the value of $\eta$, we have the conclusion.

\subsubsection{End of proof of Lemma 6.9.}

In the case where $\left|z_{2}\right| \leq\left|z_{1}\right|$, the proof goes exactly as for Lemma 6.8 (without having to take into account the value of $\frac{z_{2}}{z_{1}}+1$ ). 
When $\left|z_{1}\right| \leq\left|z_{2}\right|$, it is enough to consider the case where $\left|z_{1}\right| \geq\left|z_{2}\right|^{3 / 2}$ (Lemma 6.16 takes care of the remaining case).

Consider the analytic disk

$$
\varphi(\zeta)=\left(\frac{z_{1}}{z_{2}} \zeta, \zeta\right), \text { and } u(\zeta):=G_{\varepsilon}(\varphi(\zeta))
$$

Let $\delta_{1}<\delta$, to be chosen later. By Corollary 6.15, for $|\zeta|=\delta_{1}$,

$$
u(\zeta)=G_{\varepsilon}(\varphi(\zeta)) \leq \frac{3}{2} \log \delta_{1}+C
$$

On the other hand, if $|\zeta|=\left|\frac{z_{1}}{z_{2}}\right|^{2}$, then $\varphi(\zeta) \in\left\{w:\left|w_{1}\right| \leq\left|w_{2}\right|^{3 / 2}\right\}$ and by Lemma 6.16, for any $\eta_{1}>0$ we can choose $|\varepsilon|$ small enough so that

$$
u(\zeta) \leq 4 \log \left|\frac{z_{1}}{z_{2}}\right|+\eta_{1} .
$$

Applying the three-circle theorem for $\zeta=z_{2}$, we get

$$
\begin{aligned}
G_{\varepsilon}(z)=u\left(z_{2}\right) \leq & \frac{2 \log \left|\frac{z_{1}}{z_{2}}\right|-\log \left|z_{2}\right|}{2 \log \left|\frac{z_{1}}{z_{2}}\right|-\log \delta_{1}}\left(\frac{3}{2} \log \delta_{1}+C\right) \\
& +\frac{\log \left|z_{2}\right|-\log \delta_{1}}{2 \log \left|\frac{z_{1}}{z_{2}}\right|-\log \delta_{1}}\left(4 \log \left|\frac{z_{1}}{z_{2}}\right|+\eta_{1}\right) .
\end{aligned}
$$

Let $\eta_{2}>0$. In order to estimate the first term in the right hand side of the above inequality, we use

$$
\frac{\frac{3}{2} \log \delta_{1}+C}{2 \log \left|\frac{z_{1}}{z_{2}}\right|-\log \delta_{1}}=\frac{-\frac{3}{2}+\frac{C}{\left|\log \delta_{1}\right|}}{1+\frac{2 \log \left|\frac{z_{1}}{z_{2}}\right|}{\left|\log \delta_{1}\right|}} \leq-\frac{3}{2}+\frac{C}{\left|\log \delta_{1}\right|}
$$

for $\delta_{1}$ small enough. Notice that $2 \log \left|\frac{z_{1}}{z_{2}}\right|-\log \left|z_{2}\right|=\log \left|\frac{z_{1}^{2}}{z_{2}^{3}}\right| \geq 0$, and also $\log \left|\frac{z_{1}^{2}}{z_{2}^{3}}\right| \leq-\log \left|z_{2}\right| \leq-\log \delta$, so

$$
-\frac{3}{2}+\frac{C}{\left|\log \delta_{1}\right|} \leq-\frac{3}{2}+\frac{\eta_{2}}{|\log \delta|} \leq-\frac{3}{2}+\frac{\eta_{2}}{\log \left|\frac{z_{1}^{2}}{z_{2}^{3}}\right|}
$$


To estimate the second term in the right hand side of (6.12), it is enough to consider the case where $4 \log \left|\frac{z_{1}}{z_{2}}\right|+\eta_{1}<0$. We use

$$
\begin{gathered}
\frac{\log \left|z_{2}\right|-\log \delta_{1}}{2 \log \left|\frac{z_{1} \mid}{z_{2}}\right|-\log \delta_{1}}=\frac{1-\frac{|\log | z_{2}||}{\left|\log \delta_{1}\right|}}{1-\frac{2|\log | \frac{z_{1}}{z_{2}} \mid}{\left|\log \delta_{1}\right|}} \geq 1-\frac{|\log | z_{2}||}{\left|\log \delta_{1}\right|} \geq \\
1-\frac{\eta_{2}}{\left|4 \log \delta+\eta_{1}\right|} \geq 1-\frac{\eta_{2}}{|4 \log | \frac{z_{1}}{z_{2}}\left|+\eta_{1}\right|},
\end{gathered}
$$

for $\delta_{1}$ small enough.

Finally, (6.12) implies

$$
u\left(z_{2}\right) \leq-\frac{3}{2}\left(2 \log \left|\frac{z_{1}}{z_{2}}\right|-\log \left|z_{2}\right|\right)+\eta_{2}+4 \log \left|\frac{z_{1}}{z_{2}}\right|+\eta_{1}+\eta_{2},
$$

from which the desired estimate follows.

\section{REFERENCES}

[1] D. Barlet, Espace analytique réduit des cycles analytiques complexes compacts d'un espace analytique complexe de dimension finie, Fonctions de plusieurs variables complexes, II (Sém. François Norguet, 1974-1975), Lecture Notes in Math., Vol. 482, Springer Berlin (1975), p. 1-158

[2] E. Bedford, B. A. Taylor, A new capacity for plurisubharmonic functions, Acta Math. 149 (1982), 1-41.

[3] J. Briançon, H. Skoda, Sur la clôture intégrale d'un idéal de germes de fonctions holomorphes en un point de $\mathbb{C}^{n}$, C. R. Acad. Sci Série A 278 (1974), 949-951.

[4] M. Carlehed, J. Wiegerinck, Le cône des fonctions plurisousharmoniques négatives et une conjecture de Coman, Ann. Pol. Math. 80 (2003), 93-108.

[5] U. Cegrell, The general definition of the complex Monge-Ampère operator, Ann. Inst. Fourier (Grenoble) 54 (2004), 159-179.

[6] U. Cegrell, Weak-* convergence of Monge-Ampère measures, Math. Z. 254 (2006), 505-508.

[7] H. I. Celik, E. A. Poletsky, Fundamental solutions of the complex Monge-Ampère equation, Ann. Polon. Math. 57 (1997), no. 2, 103-110.

[8] D. Coman, The pluricomplex Green function with two poles in the unit ball of $\mathbb{C}^{n}$, Pacific J. Math. 194 (2000), 257-283.

[9] J. D'Angelo, Several Complex Variables and the Geometry of Real Hypersurfaces, Studies in Advanced Mathematics, CRC Press, Boca Raton-Ann ArborLondon-Tokyo, 1993.

[10] J.-P. Demailly, Mesures de Monge-Ampère et mesures pluriharmoniques, Math. Z. 194 (1987), 519-564.

[11] J.-P. Demailly, Complex Analytic and Differential Geometry, manuscript, Université de Grenoble I, Institut Fourier, 455 p., http://www-fourier.ujf-grenoble.fr/ demailly/books.html

[12] J.-P. Demailly, Estimates on Monge-Ampère operators derived from a local algebra inequality, in Complex Analysis and Digital Geometry, Proceedings 
from the Kiselmanfest, 2006, Uppsala University, 2009, 131-143; available at arXiv:0709.3524v2.

[13] A. Douady, Le problème des modules pour les sous-espaces analytiques compacts d'un espace analytique donné, Ann. Inst. Fourier (Grenoble) 16 (1966), fasc. $1,1-95$.

[14] A. Douady, Flatness and privilege, L'Enseignement Mathématique. Revue Internationale. IIe Série 14 (1968), 47-74.

[15] A. Edigarian, Remarks on the pluricomplex Green function, Univ. Iagel. Acta Math. 37 (1999), 159-164.

[16] L. Hörmander, An Introduction to Complex Analysis in Several Variables, Third Edition (revised), Mathematical Library, Vol. 7, North Holland, Amsterdam-New York-Oxford-Tokyo, 1990.

[17] F. Lárusson, R. Sigurdsson, Plurisubharmonic functions and analytic discs on manifolds, J. Reine Angew. Math. 501 (1998), 1-39.

[18] P. Lelong, Fonction de Green pluricomplexe et lemmes de Schwarz dans les espaces de Banach, J. Math. Pures Appl. 68 (1989), 319-347.

[19] L. Lempert, La métrique de Kobayashi et la représentation des domaines sur la boule, Bull. Soc. Math. France 109 (1981), no. 4, 427-474.

[20] J. I. Magnússon, A global morphism from the Douady space to the cycle space, Math. Scand. 101 (2007), no. 1, 19-28.

[21] H. Nakajima, Lectures on Hilbert schemes of points on surfaces, University Lecture Series vol. 18. American Mathematical Society (1999).

[22] E. A. Poletsky, Holomorphic Currents, Indiana Univ. Math. J. 42 (1993), no. $1,85-144$.

[23] E. A. Poletsky, B. V. Shabat, Invariant Metrics, in Current Problems in Mathematics. Fundamental Directions, Vol. 9, 292 (Itogi nauki i Tekhniki, Akad. Nauk SSSR, Vsesoyuz. Inst. Nachn. i Tekhn. Inform., Moscow, 1986), 73-125. English translation in Several Complex Variables III, Encyclopaedia of Mathematical Sciences, Vol. 9, Springer Verlag (1989), 63-111.

[24] A. Rashkovskii, R. Sigurdsson, Green functions with singularities along complex spaces, Int. J. Math. 16 (2005), no. 4, 333-355.

[25] P. J. Thomas, An example of limit of Lempert functions, Vietnam Journal of Mathematics, 35 (2007), no. 3, 317-330.

[26] P. J. Thomas, Nguyen Van Trao, Pluricomplex Green and Lempert functions for equally weighted poles, Arkiv för Matematik, 41 (2003), no. 2, 381-400.

[27] P. J. Thomas, Nguyen Van Trao, Convergence and multiplicities for the Lempert function, Arkiv för Matematik, 47 (2009), no. 1, 183-204.

[28] A. K. Tsikh, Multidimensional Residues and Their Applications, Translations of Mathematical Monographs, Vol. 103, American Mathematical Society, Providence, 1992.

[29] V.P. Zahariuta, Spaces of analytic functions and maximal plurisubharmonic functions. D. Sci. Dissertation, Rostov-on-Don, 1984.

[30] O. Zariski, P. Samuel, Commutative algebra. Vol. II, Reprint of the 1960 edition. Graduate Texts in Mathematics, Vol. 29. Springer-Verlag, New YorkHeidelberg, 1975. 
Jón I. Magnússon and Ragnar Sigurdsson, Science Institute, UniVERsity of ICELAND, Dunhaga 3, IS-107 REYkJAVIK, ICELAND

E-mail address: jim@hi.is, ragnar@hi.is

Alexander Rashkovskit, Faculty of Science and Technology, UniVersity of Stavanger, N-4036 Stavanger, Norway

E-mail address: alexander.rashkovskii@uis.no

Pascal J. Thomas, Université de Toulouse, UPS, insa, UT1, UTM, Institut de Mathématiques de Toulouse, F-31062 Toulouse, France

E-mail address: pthomas@math.univ-toulouse.fr 